\title{
GLOBAL INVARIANTS FOR MEASURED FOLIATIONS
}

\author{
BY
}

\author{
STEVEN HURDER
}

\begin{abstract}
New exotic invariants for measured foliations are constructed, which we call the $\mu$-classes of a pair $(\mathcal{F}, \mu)$. The dependence of the $\mu$-classes on the geometry of the foliation $\mathcal{F}$ is examined, and the dynamics of a foliation is shown to determine the $\mu$-classes in many cases. We use the $\mu$-classes to study the classifying space $B \Gamma_{S L_{q}}$ of foliations with a transverse invariant volume form, and we show the homotopy groups of $B \Gamma_{S L_{q}}$ are uncountably generated starting in degrees $q+3$. New invariants for groups of volume preserving diffeomorphisms also arise from the $\mu$-classes; these invariants are nontrivial and related to the geometric aspects of the group action. Relations between the $\mu$-classes and the secondary classes of a foliation are exhibited.
\end{abstract}

0. Introduction. In this paper we construct new exotic characteristic classes for foliations which have a transverse invariant measure. These invariants, which we call the $\mu$-classes, are similar to the secondary classes and take values in the real cohomology $H^{*}(M)$ of the foliated manifold $M$ (Theorem 1.6). The values of the $\mu$-classes are strongly related to the geometry of the foliation (Proposition 3.4). The $\mu$-classes have applications to the study of foliations with a transverse invariant volume form (Theorems 5.1 and 5.2), to the computations of the homology groups of the discrete group of volume preserving diffeomorphisms of $\mathbf{R}^{n}$ (Theorem 7.4), and to the problem of relating the secondary classes of a foliation with its global geometry $[15,20]$.

The $\mu$-classes arose from an attempt to obtain general invariants of a foliation which are intermediate between the leaf and secondary classes. They are a special case of a family of universal invariants associated to a $C^{2}$-foliation, the Weil operators. In codimension one, the introduction of the Weil operator is a decisive ingredient in Duminy's elegant proof of the Sullivan conjecture about the Godbillon-Vey class [9]. The properties of the Weil operators in higher codimensions are given in [15] where they are used to study the "residuable" secondary classes. We briefly describe the construction of the Weil operators and their relations with the $\mu$-classes and other foliation invariants in $\$ 2$. For a foliation with all leaves compact we note that the Weil operators are identically zero (Proposition 2.6).

We denote by $\mathscr{F}$ a $C^{2}$-foliation of codimension $q$ on a paracompact manifold $M$, and $\mu$ denotes a transverse invariant measure for $\mathscr{F}$. Recall that $\mu$ assigns to each

Received by the editors June 28, 1982 and, in revised form, November 9, 1982.

1980 Mathematics Subject Classification. Primary 57R30, 57R20, 58F11; Secondary 58H10, $28 \mathrm{D} 15$.

Key words and phrases. Foliations, invariant measures, characteristic classes, group homology, geometry of foliations. 
compact transversal $T \subseteq M$ a finite number $\mu(T)$. We do not require $\mu$ to be positive; that is, $\mu(T)$ need not be positive. For the flow $\phi_{t}: M \rightarrow M$ of a vector field $X$ on $M$ tangent to $\mathscr{F}$, the invariance of $\mu$ implies $\mu\left(\phi_{t} T\right)=\mu(T)$. There is an extensive literature giving methods for constructing transverse invariant measures and applications $[\mathbf{1 6}, \mathbf{2 9}, \mathbf{3 0}, \mathbf{3 1}, \mathbf{3 5}]$. The pair $(\mathscr{F}, \mu)$ is said to be a measured foliation on $M$.

One definition of the $\mu$-classes of a measured foliation $(\mathscr{F}, \mu)$ is that they are the integral of the leaf classes of $\mathscr{F}$ over the generalized measure space $(M / \mathscr{F}, \mu)$. Since the quotient $M / \mathcal{F}$ is genericly a singular, nonhausdorff space, this integral must be interpreted via Connes' theory of noncommutative integration [7]. This theory was used by Connes to prove the index theorem for foliations [8]. The index invariants are defined by integrating tangent data to $\mathscr{F}$ (the Euler and Pontrjagin classes of the subbundle $\mathscr{F} \subseteq T M)$ over $(M / \mathscr{F}, \mu)$. In contrast, the $\mu$-classes are defined by integrating normal data to $\mathscr{F}$ over $(M / \mathscr{F}, \mu)$. The normal data consists of the differential invariants associated to the canonical flat structure on the normal bundle to $\mathscr{F}$. We note, however, that the construction given in $\$ 1$ below for the $\mu$-classes is direct, and does not require the theory of noncommutative integration.

The organization of the rest of this paper is as follows. Part I defines the $\mu$-classes and studies their properties. $§ 1$ gives background material and then constructs the $\mu$-classes. The Weil operators are defined in $\$ 2$ and we derive the $\mu$-classes from them. Relations between the geometry of the foliation and the values of the $\mu$-classes are examined in $\S 3$. This yields a convenient method for producing examples with nonvanishing $\mu$-classes (Proposition 3.4). Several examples are described in \$4. Part II studies $S L_{q}$-foliations, where $\mu$ is represented by a smooth, closed nonvanishing $q$-form. The $\mu$-classes have corresponding universal classes in the cohomology of the Haefliger classifying space $B \Gamma_{S L_{q}}$. This is pursued in $\$ 5$, where we show many of the $\mu$-classes inject into $H^{*}\left(B \Gamma_{S L_{q}}\right)$ and are independently variable. Extensive nonvanishing results are also given for $H^{*}\left(B \bar{\Gamma}_{S L_{q}}\right)$. $\$ 6$ examines the rational homotopy groups $\pi_{*}\left(B \bar{\Gamma}_{S L_{a}}\right) \otimes \mathbf{Q}$. This information is used in $\S 7$ to calculate part of the group homology $H_{m}\left(B \overline{\operatorname{Diff}}_{\omega}^{c} \mathbf{R}^{q} ; \mathbf{Q}\right)$, where $\omega$ is any volume form with infinite total volume. In particular, we show $H_{3}\left(B \overline{\operatorname{Diff}}_{\omega}^{c} \mathbf{R}^{q} ; \mathbf{Q}\right)$ has uncountable dimension for $q \geqslant 3$ so the vanishing result of Thurston-Banyaga that $H_{1}\left(B \overline{\operatorname{Diff}}_{\omega}^{c} \mathbf{R}^{q} ; Z\right)=0$ is almost the best possible.

It is a pleasure to thank D. McDuff, A. Haefliger and C. Lazarov for stimulating conversations during the progress of this work. The material in $\$ 2$ was developed in discussions with J. Heitsch and P. Schweitzer. The author is grateful to the Institute for Advanced Study for its hospitality and support.

\section{I. $\mu$-CLASSES AND MEASURED FOLIATIONS}

1. Transverse measures and $\mu$-classes. We assume throughout that $M$ is an oriented manifold, $(\mathscr{F}, \mu)$ is a measured foliation on $M$, and either $M$ is compact or the measure $\mu$ is absolutely continuous. In the latter case, $\mu$ can be represented by a $q$-form $\omega$ with locally integrable coefficients so that for a transversal $T, \mu(T)=\int_{T} \omega$. The $q$-form $\omega$ satisfies $i(X) \omega=0=L_{X} \omega$ for all vector fields $X$ tangent to $\mathscr{F}$, and thus has distributional derivative $d \omega=0$. 
A foliation cycle $z$ defines a transverse invariant measure $\mu_{z}$ via "counting intersection points" [35]. For a compact leaf $L \subseteq M$ and oriented transversal $T$ in general position with respect to $L$, set

$$
\mu_{L}(T)=\#(L \cap T)=\text { number of points of intersection. }
$$

For $T$ compact, $\mu_{L}(T)$ is clearly finite; $\mu_{L}$ is easily seen to be invariant and has support on the leaf $L$. More generally, a leaf of subexponential growth in a compact manifold determines a foliation cycle and an invariant measure $\mu_{L}[20,35]$.

An invariant measure $\mu$ with compact support determines a closed current $c_{\mu}$ on $M$. We recall from [31] the definition of $c_{\mu}$ : Let dimension $M=m$ and $Q(M)$ denote the de Rham complex. Choose a finite open cover $\left\{U_{1}, \ldots, U_{N}\right\}$ of supp $\mu$ by foliation charts, where $\phi_{i}: U_{i} \rightarrow D^{m-q} \times D^{q} \subseteq \mathbf{R}^{m}$ identifies the leaves of $\left.\mathscr{F}\right|_{U_{i}}$ with the plaques $D^{m-q} \times\{p t\}$. Let $\left\{\lambda_{1}, \ldots, \lambda_{N+1}\right\}$ be a partition of unity subordinate to the open cover $\left\{U_{1}, \ldots, U_{N}, M\right.$-supp $\left.(\mu)\right\}$ of $M$. The definition of the current $c_{\mu}$ : $\mathbb{Q}^{m-q}(M) \rightarrow \mathbf{R}$ is now given by, for a form $\eta \in \mathbb{Q}^{m-q}(M)$ :

$$
c_{\mu}(\eta)=\sum_{i=1}^{N} \int_{D^{q}}\left\{\int_{D^{m-q}} \lambda_{i} \eta\right\} \mu .
$$

The value of $c_{\mu}(\eta)$ can be shown to be independent of the choices made [31]. Further, if $\eta$ is an exact form then $c_{\mu}(\eta)=0$, so $c_{\mu}$ is a closed current.

For a compact manifold $M$, possibly with boundary, and a closed current $c$ : $Q^{n}(M) \rightarrow \mathbf{R}$, there is a corresponding (dual) cohomology class $\left[c^{*}\right] \in H^{m-n}(M)$. The class dual to $c_{\mu}$ above is denoted $[\mu] \in H^{q}(M)$.

Leaf and secondary classes. Let $Q$ denote the normal bundle to $\mathscr{F}$ in $T M$ and $\pi$ : $T M \rightarrow Q$ the projection. A connection $\nabla$ on $Q$ is said to be a Bott connection [4] if $\nabla_{X} \pi(Y)=\pi\left(L_{X} Y\right)$ for all vector fields $X$ tangent to $\mathscr{F}$ and $Y$ normal to $\mathscr{F}$. The Jacobi identity for vector fields implies a Bott connection restricts to a flat connection on the normal bundle to a leaf, $\left.Q\right|_{L} \rightarrow L$.

The choice of a Bott connection for $\mathscr{F}$ defines a differential graded algebra (dga) map $\Delta: W O_{q} \rightarrow \mathbb{Q}(M)$. The algebra $W O_{q}$ is the product of an exterior algebra with a polynomial algebra truncated in degrees above $2 q$,

$$
W O_{q}=\Lambda\left(y_{1}, y_{3}, \ldots, y_{q^{\prime}}\right) \otimes \mathbf{R}\left[c_{1}, \ldots, c_{q}\right]_{q},
$$

where $q^{\prime}=2[(q+1) / 2]-1$, degree $y_{i}=2 i-1$, degree $c_{i}=2 i$ and the differential is determined by $d y_{i}=c_{i}, d c_{i}=0$. The construction of $\Delta$ and its properties are described in detail in the foundational papers of Bott and Haefliger [4,6], and from the Chern-Weil point of view by Kamber and Tondeur $\left[22,38\right.$. The image of $\Delta_{*}$ : $H^{*}\left(W O_{q}\right) \rightarrow H^{*}(M)$ consists of the secondary classes of $\mathscr{F}$.

If the normal bundle to $\mathscr{F}$ is trivial then a choice of framing of $Q$, given by a section $s: M \rightarrow P$ of the associated frame bundle, determines an extension of $\Delta$ to a dga map $\Delta^{s}: W_{q} \rightarrow \mathbb{Q}(M)$, where

$$
W_{q}=\Lambda\left(y_{1}, y_{2}, \ldots, Y_{q}\right) \otimes \mathbf{R}\left[c_{1}, \ldots, c_{q}\right]_{q}
$$

with degree $y_{i}=2 i-1$, and $d y_{i}=c_{i}$ again. We remark that if the previous $\Delta$ is now constructed using the metric for which the framing $s$ is orthonormal, then $\Delta^{s}$ restricts 
to $\Delta$ on $W O_{q}$. The image of $\Delta_{*}^{s}$ consists of the secondary classes for the framed foliation $(\mathcal{F}, s)$.

Next recall the definition of the leaf classes for a leaf $L \subseteq M$. Let $\Gamma\left(M, Q^{*}\right) \subseteq$ $\mathbb{Q}^{\prime}(M)$ denote the space of 1 -forms which annihilate $\mathscr{F}$, and $\Gamma\left(M, \Lambda^{i} Q^{*}\right)$ the sections of the $i$ th exterior power bundle. The crucial property of the Chern-Weil homomorphism $\Delta$ constructed using a Bott connection is that for each Chern polynomial $c_{i}$,

$$
\Delta^{s}\left(c_{i}\right)=\Delta\left(c_{i}\right) \in \Gamma\left(M, \Lambda^{i} Q^{*}\right) \wedge \mathbb{Q}^{i}(M) .
$$

This implies $\Delta\left(c_{i}\right)$ vanishes when restricted to the leaf $L$, so each $\left.\Delta\left(y_{i}\right)\right|_{L}$ or $\left.\Delta^{s}\left(y_{i}\right)\right|_{L}$ is a closed form which is an invariant of the flat connection on $\left.Q\right|_{L} \rightarrow L$.

For an index $I=\left(i_{1}, \ldots, i_{r}\right)$ with $1 \leqslant i_{1}<\cdots<i_{r} \leqslant q$, set $y_{I}=y_{i_{1}} \cdots y_{i_{r}} \in W_{q}$. If all $i$ are odd then $y_{I} \in W O_{q}$. The form $\Delta\left(y_{I}\right)$ or $\Delta^{s}\left(y_{I}\right) \in \mathbb{Q}(M)$ is not closed in general, but the above remarks imply $\left.\Delta\left(y_{I}\right)\right|_{L}$ is closed and determines a class in $H^{*}(L)$. The restrictions of $\Delta$ or $\Delta^{s}$ thus define graded algebra maps

$$
\chi_{L}: H^{*}\left(g l_{q}, O_{q}\right) \rightarrow H^{*}(L), \quad \chi_{L}^{s}: H^{*}\left(g l_{q}\right) \rightarrow H^{*}(L) .
$$

Here, we identify $H^{*}\left(g l_{q}\right)$ with $\Lambda\left(y_{1}, \ldots, y_{q}\right)$ endowed with the trivial differential, and similarly for the relative Lie algebra cohomology $H^{*}\left(g l_{q}, O_{q}\right)$. The image of $\chi_{L}$ consists of the leaf classes of $\mathscr{F}$ for $L$.

The map $\chi_{L}$ is an invariant of the germ of $\mathscr{F}$ about $L$, and in fact depends only on the flat bundle structure of $\left.Q\right|_{L} \rightarrow L$. To be precise, recall that the foliation in a neighborhood of $L$ determines the linear holonomy $d h: \pi_{1}(L, x) \rightarrow G l_{q} \mathbf{R}$, for $x \in L$ a basepoint. The flat structure on $\left.Q\right|_{L}$ is classified by the induced map $B(d h)$ : $L \rightarrow B G l_{q}^{\delta} \mathbf{R}$, where $G l_{q}^{\delta} \mathbf{R}$ has the discrete topology. Shulman and Tischler prove in [33] the

Proposition 1.3. There is a commutative diagram

$$
\begin{array}{ccc}
H^{*}\left(g l_{q}, O_{q}\right) & \stackrel{\chi_{L}}{\rightarrow} & H^{*}(L) \\
V E \searrow & & \nearrow B(d h)^{*} \\
& H^{*}\left(B G l_{q}^{\delta} \mathbf{R}\right) &
\end{array}
$$

where VE is the van Est map defining the differentiable cohomology of $G l_{q}^{\delta} \mathbf{R}$.

If $Q$ is a trivial bundle, then the classifying map $M \rightarrow B G l_{q} \mathbf{R}$ is trivial, so the composition $L \rightarrow B G l_{q}^{\delta} \mathbf{R} \rightarrow B G l_{q} \mathbf{R}$ is also homotopic to the constant map. The choice of a framing $s$ of $Q$ defines a lift $B(d h, s): L \rightarrow B \overline{G l} \mathbf{R}$, where $\overline{G l} l_{q} \mathbf{R}$ is the homotopy fiber of the inclusion $G l_{q}^{\delta} \mathbf{R} \subseteq G l_{q} \mathbf{R}$. The analog of Proposition 1.3 is then

Proposition 1.4. There is a commutative diagram:

$$
\begin{array}{ccc}
H^{*}\left(g l_{q}\right) & \stackrel{\chi_{L}^{s}}{\rightarrow} & H^{*}(L) \\
V E \searrow & & \nearrow B(d h, s)^{*} \\
& H^{*}\left(B \overline{G l_{q}} \mathbf{R}\right) &
\end{array}
$$

REMARK 1.5. Note that if the linear holonomy of $L$ is trivial, or $B(d h)^{*}$ is the zero map, then 1.3 implies $\chi_{L}$ is the zero map. However, even if $L$ has trivial holonomy, 
with the proper choice of framing of $Q$ one can sometimes make $\chi_{L}^{s}$ nontrivial. Of course, $\chi_{L}^{s}$ must still vanish in this case when restricted to $H^{*}\left(g l_{q}, O_{q}\right) \subseteq H^{*}\left(g l_{q}\right)$.

The $\mu$-classes. We say two measured foliations $\left(\mathscr{F}_{0}, \mu_{0}\right)$ and $\left(\mathscr{F}_{1}, \mu_{1}\right)$ on $M$ are measured concordant if there is a codimension $q$ measured foliation $(\mathscr{F}, \mu)$ on $M \times[0,1]$ which restricts to $\left(\mathscr{F}_{t}, \mu_{t}\right)$ on $M \times\{t\}$ for $t=0$ or 1 .

THeOREM 1.6. Let $(\mathscr{F}, \mu)$ be a measured foliation on $M$ and assume that either $M$ is compact and without boundary, or that $\mu$ is absolutely continuous.

(a) There is a characteristic map

$$
\chi_{\mu}: H^{*}\left(g l_{q}, O_{q}\right) \rightarrow H^{*+q}(M)
$$

raising degree by $q$, which depends only on the measured concordance class of $(\mathscr{F}, \mu)$ and is functorial with respect to pull-backs.

(b) Given a framing $s$ of the normal bundle to $\mathcal{F}$, there is a characteristic map

$$
\chi_{\mu}^{s}: H^{*}\left(g l_{q}\right) \rightarrow H^{*+q}(M)
$$

raising degree by $q$, which depends only on the measured concordance class of $(\mathscr{F}, \mu)$ and the homotopy class of the framing $s$.

REMARKS . 1.7. $\chi_{\mu}$ and $\chi_{\mu}^{s}$ are homomorphisms of real vector spaces, but do not preserve the algebra structures.

1.8. The class $\chi_{\mu}(1) \in H^{q}(M)$ is the canonical class $[\mu]$ associated to the measure.

1.9. If $\partial M \neq \varnothing$ and $\left.\mathscr{F}\right|_{\partial M}$ has codimension $(q-1)$, then one can also define maps $\chi_{\mu}, \chi_{\mu}^{s}$ with range $H^{*}(M, \partial M)$.

1.10. If the measure $\mu$ is represented by a smooth, nonvanishing $q$-form $\omega$, then $(\mathscr{F}, \mu)$ is an $S L_{q}$-foliation and 1.6 yields new differential invariants.

Proof of 1.6. We assume $(\mathscr{F}, \mu)$ is given, a choice of Bott connection for $\mathscr{F}$ has been made and for part (b) that a framing $s$ is given. From this data $\chi_{\mu}$ and $\chi_{\mu}^{s}$ will be defined. It is then standard to show the independence of the maps from the choices involved.

First, assume $\mu$ is represented by a $q$-form $\omega$ which has distributional derivative $d \omega=0$. For $y_{I}=y_{i_{1}} \wedge \cdots \wedge y_{i_{r}}$ of degree $p$, set

$$
\chi_{\mu}\left(y_{I}\right)=\left[\Delta\left(y_{I}\right) \wedge \omega\right] \in H^{p+q}(M) .
$$

Note that this is well defined:

$$
d\left(\Delta\left(y_{I}\right) \wedge \omega\right)=d \Delta\left(y_{I}\right) \wedge \omega+(-1)^{p} \Delta\left(y_{I}\right) \wedge d \omega=0
$$

since $d \Delta\left(y_{I}\right) \in \Gamma\left(M, Q^{*}\right) \wedge \mathbb{Q}^{P}(M)$, and this ideal is annihilated by exterior product with $\omega$. The monomials $\left\{y_{I} \in W O_{q}\right\}$ are a basis for $H^{*}\left(g l_{q}, O_{q}\right)$ so extend by linearity to obtain $\chi_{\mu}$. The construction of $\chi_{\mu}^{s}$ is entirely analogous, where for $y_{I} \in W_{q}$ we set

$$
\chi_{\mu}^{s}\left(y_{I}\right)=\left[\Delta^{s}\left(y_{I}\right) \wedge \omega\right] \in H^{p+q}(M) .
$$

Next, suppose $\mu$ is an arbitrary transverse invariant measure and $M$ is closed. For $y_{I}$ of degree $p$, we first define a closed current

$$
\tilde{\chi}_{\mu}\left(y_{I}\right): \mathbb{Q}^{m-p-q}(M) \rightarrow \mathbf{R} .
$$


Given a form $\eta$ of degree $m-p-q$, set $\tilde{\chi}_{\mu}\left(y_{I}\right)(\eta)=c_{\mu}\left(\Delta\left(y_{I}\right) \wedge \eta\right)$ where $c_{\mu}$ is the closed current associated to $\mu$ in (1.1). We must show $\tilde{\chi}_{\mu}\left(y_{I}\right)$ is closed. For this it suffices to assume $\eta=d \zeta$ where $\zeta$ has support in an open foliation chart $\phi$ : $U \rightarrow D^{m-q} \times D^{q}$. Then

$$
\begin{aligned}
\tilde{\chi}_{\mu}\left(y_{I}\right)(d \zeta) & =c_{\mu}\left(\Delta\left(y_{I}\right) \wedge d \zeta\right) \\
& = \pm c_{\mu}\left(d\left\{\Delta\left(y_{I}\right) \wedge \zeta\right\}\right) \pm c_{\mu}\left(d \Delta\left(y_{I}\right) \wedge \zeta\right) \\
& = \pm c_{\mu}\left(d \Delta\left(y_{I}\right) \wedge \zeta\right) \\
& = \pm \int_{a \in D^{q}}\left\{\int_{D^{m-q} \times\{a\}} d \Delta\left(y_{I}\right) \wedge \zeta\right\} \mu \\
& =0
\end{aligned}
$$

because $\Delta\left(y_{I}\right)$ restricted to any leaf is closed, and $\left.d \Delta\left(y_{I}\right)\right|_{D^{m-4} \times\{a\}}=0$ in particular.

$M$ is assumed to be closed and oriented, so we can define $\chi_{\mu}\left(y_{I}\right) \in H^{p+q}(M)$ to be the dual class to the closed current $\tilde{\chi}_{\mu}\left(y_{I}\right)$. Then we extend by linearity to obtain the characteristic map $\chi_{\mu}$. The construction of $\chi_{\mu}^{s}$ is similar.

The independence of $\chi_{\mu}$ and $\chi_{\mu}^{s}$ from the choice of Bott connection, their dependence only on the measured concordance class of $(\mathscr{F}, \mu)$ and the dependence of $\chi_{\mu}^{s}$ only on the homotopy class of $s$ are all proved in the same manner. We prove just the first of these claims and leave the rest to the reader.

Let $\nabla^{0}, \nabla^{1}$ be two Bott connections for $\mathscr{F}$. The projection $M \times[0,1] \rightarrow M$ pulls $(\mathscr{F}, \mu)$ back to a measured foliation $\left(\mathcal{F}^{\prime}, \mu^{\prime}\right)$, and a Bott connection for $\mathscr{F}^{\prime}$ is given by $\nabla^{t}=(1-t) \nabla^{0}+t \nabla^{1}$ where $t$ is the coordinate in $[0,1]$. Let $\chi_{\mu^{\prime}}: H^{*}\left(g l_{q}, O_{q}\right) \rightarrow$ $H^{*}(M \times I)$ be the characteristic map defined using $\nabla^{t}$. For the two inclusions $\zeta_{t}$ : $M \times\{t\} \rightarrow M \times[0,1], t=0$ or 1 , we note that $\zeta_{t}^{*} \circ \chi_{\mu^{\prime}}$ is the characteristic map of $(\mathcal{F}, \mu)$ calculated using the Bott connection $\nabla^{t}$. Since $\zeta_{0} \simeq \zeta_{1}, \zeta_{0}^{*}=\zeta_{1}^{*}$ so our conclusion follows.

2. The Weil operators. The leaf, secondary and $\mu$-classes of a foliation $\mathscr{F}$ are special cases of a very general set of invariants for $\mathscr{F}$, which we call the Weil operators in reference to that fact that they are the transgressive operators in the Chern-Weil theory of characteristic classes. We briefly describe their construction and relevance to the standard invariants of $\mathscr{F}$, which helps to show why certain properties of the $\mu$-classes carry over to the secondary classes. The Weil operators were first introduced in the context of codimension one foliations by Duminy [9], where there is just one, corresponding to $y_{1} \in W_{1}$. A complete development and study of the Weil operators in all codimensions is given in [15]. The Weil operators are also related to the derived classes in sheaf cohomology of Kamber and Tondeur, developed in $\$ \S 6$ to 8 of [38].

We define a subcomplex $\mathbb{Q}(M ; \mathcal{F}) \subseteq \mathbb{Q}(M)$. Let $\left\{\omega_{1}, \ldots, \omega_{q}\right\}$ be a (local) set of defining 1-forms for $\mathscr{F}$. Set

$$
\mathbb{Q}(M ; \mathcal{F})=\left\{\phi \in \mathbb{Q}(M) \mid \phi \wedge \omega_{i}=0 \text { for } 1 \leqslant i \leqslant q\right\} .
$$

By integrability, $d \omega_{i}=\sum_{j=1}^{q} \omega_{i j} \wedge \omega_{j}$ for 1-forms $\left(\omega_{i j}\right)$, and so $0=d\left(\phi \wedge \omega_{i}\right)=d \phi$ $\wedge \omega_{i} \pm \phi \wedge d \omega_{i}=d \phi \wedge \omega_{i}$. Thus $\mathbb{Q}(M ; \mathscr{F})$ is a differential subcomplex, and $H^{*}(M, \mathcal{F})$ denotes its cohomology. 
In the space of currents $C_{p}(M)$ on $M$, define a subcomplex, for $p \geqslant 0$

$$
C_{p}(M ; \mathscr{F})=\left\{c \in C_{p}(M) \text { such that } c \mid \omega_{i} \cdot \mathscr{Q}(M)=0,1 \leqslant i \leqslant q\right\} .
$$

This is a differential subcomplex, and $H_{*}(M, \mathscr{F})$ denotes its homology.

EXAMPLE 2.1. A transverse invariant measure $\mu$ with compact support defines an element $\left[c_{\mu}\right] \in H_{n-q}(M, \mathscr{F})$. If $\mu$ is represented by a closed nonzero $q$-form $\omega$, then we have a class $[\omega] \in H^{q}(M, \mathscr{F})$, and $[\omega] \neq 0$ since $Q^{q-1}(M ; \mathscr{F})=0$.

EXAMPLE 2.2. A Chern polynomial $c_{J} \in W O_{q}$ of degree $2 q$ satisfies $\Delta\left(c_{J}\right) \in$ $\Gamma\left(M, \Lambda^{q} Q^{*}\right) \wedge \mathbb{Q}^{q}(M)$ hence determines $\left[c_{J}\right] \in H^{2 q}(M, \mathscr{F})$. Heitsch has shown the class $\left[c_{J}\right]$ is independent of the choice of the Bott connection [15].

EXAMPLE 2.3. Let $j: L \rightarrow M$ be the inclusion of any leaf of $\mathscr{F}$. Then there is a natural map $j_{*}: H_{*}(L) \rightarrow H_{*}(M, \mathscr{F})$. For any cycle $z$ in $L$, the image $j(z)$ is a foliation cycle in $M$ with compact support.

We now define the Weil operators. These were first introduced by Duminy [9] in codimension 1; the natural extension to higher codimensions is further studied in joint work with J. Heitsch [15].

THEOREM 2.4. Let $\mathcal{F}$ be a codimension $q, C^{2}$-foliation on $M$.

(a) There is a well-defined linear map for each $p \geqslant 0$

$$
\chi: H^{p}\left(g l_{q}, O_{q}\right) \rightarrow \operatorname{Hom}\left(H^{*}(M, \mathscr{F}), H^{*+p}(M)\right) .
$$

(b) If $s$ is a framing of the normal bundle to $\mathscr{F}$, then there is a linear map for each $p \geqslant 0$

$$
\chi^{s}: H^{p}\left(g l_{q}\right) \rightarrow \operatorname{Hom}\left(H^{*}(M, \mathscr{F}), H^{*+p}(M)\right) .
$$

Note the space $H^{*}(M, \mathscr{F})$ is not invariant under concordances of $\mathscr{F}$, so the Weil operators are not concordance invariants.

Proof of 2.4. For a monomial $y_{I} \in H^{p}\left(g l_{q}, O_{q}\right)$ we define $\chi\left(y_{I}\right): H^{l}(M, \mathscr{F}) \rightarrow$ $H^{l+p}(M)$ as follows: given a closed $\phi \in \mathscr{Q}(M ; \mathscr{F})$, set $\chi\left(y_{I}\right)[\phi]=\left[\Delta\left(y_{I}\right) \wedge \phi\right]$ where $\Delta: W O_{q} \rightarrow \mathbb{Q}(M)$ is the secondary map of $\S 1$. We must check that ior $\phi, \phi^{\prime} \in \mathbb{Q}(M ; \mathscr{F})$ and $\eta \in \mathbb{Q}^{\prime-1}(M ; \mathscr{F})$ with $\phi-\phi^{\prime}=d \eta$, the cohomology class $\left[\Delta\left(y_{I}\right) \phi\right]=\left[\Delta\left(y_{I}\right) \phi^{\prime}\right]$. But $d \Delta\left(y_{I}\right) \wedge \eta=0$ so

$$
\left\{\Delta\left(y_{I}\right) \wedge \phi-\Delta\left(y_{I}\right) \wedge \phi^{\prime}\right\}=d\left\{\Delta\left(y_{I}\right) \wedge \eta\right\} .
$$

The proof that $\chi\left(y_{I}\right)$ does not depend on the choice of Bott connection used to define $\Delta$ is the same as in the proof of Theorem 1.6.

The construction of $\chi^{s}$ is made using the characteristic map $\Delta^{s}$ of the framed foliation $(\mathscr{F}, s)$, but is otherwise the same as the above.

THEOREM 2.5. Let $\mathscr{F}$ be a $C^{2}$-foliation of codimension $q$ on a compact manifold $M$ without boundary.

(a) There is a well-defined linear map for each $p \geqslant 0$

$$
\tilde{\chi}: H^{p}\left(g l_{q}, O_{q}\right) \rightarrow \operatorname{Hom}\left(H_{*}(M, \mathscr{F}), H_{*-p}(M)\right) .
$$

(b) If $s$ is a framing of the normal bundle $\mathscr{F}$, then there is a linear map for each $p \geqslant 0$

$$
\tilde{\chi}^{s}: H^{p}\left(g l_{q}\right) \rightarrow \operatorname{Hom}\left(H_{*}(M, \mathscr{F}), H_{*-p}(M)\right) .
$$


Proof. For a monomial $y_{I} \in H^{p}\left(g l_{q}, O_{q}\right)$ and a closed current $[c] \in H_{l}(M, \mathcal{F})$ we define the closed current $\tilde{\chi}\left(y_{I}\right)(c): \mathbb{Q}^{l-p}(M) \rightarrow \mathbf{R}$ as follows: for $\eta \in \mathbb{Q}^{l-p}(M)$ set $\tilde{\chi}\left(y_{I}\right)(c)(\eta)=c\left(\Delta\left(y_{I}\right) \wedge \eta\right)$. To see that $\chi\left(y_{I}\right)(c)$ is closed one proceeds along the lines of (1.11), and the independence of the resulting class $\tilde{\chi}\left(y_{I}\right)[c] \in H_{l-p}(M)$ from the choice of Bott connection follows similarly.

It is apparent from the definitions of $\chi$ and $\chi^{s}$ that many of the standard foliation invariants can be recovered from them. We make these relationships precise.

Given a measured foliation ( $\mathscr{F}, \mu$ ) on $M$ with $\mu$ represented by a closed $q$-form $\omega$, we have $[\omega] \in H^{q}(M, \mathscr{F})$. The $\mu$-classes result from applying the Weil operators to this element: for $y_{I} \in H^{*}\left(g l_{q}, O_{q}\right), \chi_{\mu}\left(y_{I}\right)=\chi\left(y_{I}\right)[\omega]$. If $M$ is compact without boundary, then $\left[c_{\mu}\right] \in H_{m-q}(M, \mathscr{F})$ and the current definition of the $\mu$-classes yields $\tilde{\chi}_{\mu}\left(y_{I}\right)=\tilde{\chi}\left(y_{I}\right)\left[c_{\mu}\right]$.

A cocycle $y_{I} c_{J} \in W O_{q}$ with degree $c_{J}=2 q$ is said to be a residuable secondary class (see Heitsch [14] or Hurder [20]). For any $C^{2}$-foliation $\mathscr{F}$ and Chern polynomial $c_{J}$ of degree $2 q$ we observed in Remark 2.2 that there is a class $\left[c_{J}\right] \in H^{2 q}(M, \mathscr{F})$. The residuable secondary class $\Delta_{*}\left(y_{I} c_{J}\right)$ is obtained by applying the Weil operator $\chi\left(y_{I}\right)$ to $\left[c_{J}\right]$. From the point of view of Duminy this says the residuable secondary classes can be factored into two parts: the first, $\chi\left(y_{I}\right)$, takes values in a space of homomorphisms and the second, $\left[c_{J}\right]$, is a "point" in the space $H^{*}(M, \mathscr{F})$. For codimension $q=1$, in Duminy's terminology $\chi\left(y_{1}\right)=g$ is the Godbillon operator and $\left[c_{1}\right]=v$ is the Vey element, and one has the functional equation

$$
G V(\mathscr{F})=\Delta_{*}\left(y_{1} c_{1}\right)=\chi\left(y_{1}\right)\left[c_{1}\right]=g \circ v .
$$

The justification for this approach is the brilliant discovery by Duminy that the invariant $\chi\left(y_{1}\right)$ can be related in codimension one to the geometry of $\mathscr{F}$. He shows that $\chi\left(y_{1}\right)$ is the zero operator if $M$ is compact and $\mathscr{F}$ has no resilient leaves, and thus the Godbillon-Vey class $\Delta_{*}\left(y_{1} c_{1}\right)=\chi\left(y_{1}\right)\left[c_{1}\right]$ vanishes under the same hypothesis. This suggests that to relate the geometry of $\mathscr{F}$ with the secondary classes in codimension greater than one, a possible first step is to understand the properties of the operators $\chi\left(y_{I}\right)$.

In a previous paper (Hurder [20]) it was shown that the residuable classes for a foliation with all leaves compact must vanish. An examination of the proof of this shows that a more general result is true:

Proposition 2.6. Let $\mathcal{F}$ be a $C^{2}$-foliation of codimension $q$ on a compact manifold $M$ with all leaves of $F$ compact. Then the characteristic map

$$
\chi: H^{l}\left(g l_{q}, O_{q}\right) \rightarrow \operatorname{Hom}\left(H^{*}(M, \mathscr{F}), H^{*+l}(M)\right)
$$

is zero for all $l>0$.

Let $j: L \rightarrow M$ be the inclusion of a leaf of $\mathscr{F}$ and assume $M$ is closed. For $y_{I} \in H^{l}\left(g l_{q}, O_{q}\right)$, the linear function given by the composition

$$
H_{l}(L) \stackrel{j_{*}}{\rightarrow} H_{l}(M, \mathscr{F}) \stackrel{\tilde{\chi}\left(y_{l}\right)}{\rightarrow} H_{0}(M) \cong \mathbf{R}
$$

defines a cohomology class $\left[\tilde{\chi}\left(y_{I}\right) \circ j_{*}\right]^{*} \in H^{l}(L)$. Tracing through the various definitions one sees this is the class of the cocycle obtained by restricting $\Delta\left(y_{I}\right)$ to $L$, 
hence is the leaf class $\chi_{L}\left(y_{I}\right)=\left[\tilde{\chi}\left(y_{I}\right) \circ j_{*}\right]^{*}$. The Weil operators are thus seen to yield the leaf classes of $\mathscr{F}$ via restriction; in this sense, they are universal leaf classes for $\mathscr{F}$. This relationship also shows that the map $j_{*}: H_{*}(L) \rightarrow H_{*}(M, \mathscr{F})$ can be nonzero. Let $z \in H_{l}(L)$ and suppose there is a leaf class $\chi_{L}(y)$ which evaluates nontrivially on $z$; then $j_{*}(z) \neq 0$.

The homomorphism $\Delta_{*}$ of Chern-Weil theory is the composition of two maps, $H^{*}\left(g l_{q}, O_{q}\right) \rightarrow^{k *} H^{*}\left(P / O_{q}\right) \rightarrow H^{*}(M)$, where $P \rightarrow M$ is the $G l_{q}$-frame bundle of $Q$, and $k\left(y_{i}\right)$ transgresses to the Chern class $\Delta\left(c_{i}\right) \in \mathbb{Q}(M)$. We remark that each Weil operator $\chi\left(y_{I}\right)$ can be viewed as an "operation" in the spectral sequence for the algebraic fibration

$$
\mathcal{Q}(M, \mathscr{F}) \rightarrow \mathcal{Q}(M, \mathscr{F}) \otimes H^{*}\left(g l_{q}, O_{q}\right) \rightarrow H^{*}\left(g l_{q}, O_{q}\right),
$$

the operation transforming basic classes in $H^{*}(M, \mathcal{F})$ into classes in

$$
H^{*}\left(\mathbb{Q}(M, \mathscr{F}) \otimes H^{*}\left(g l_{q}, O_{q}\right)\right),
$$

which can then be mapped to $H^{*}(M)$. This operation in (2.7) is related to an edge map in hypercohomology defined by Kamber and Tondeur [38].

3. Geometry of the $\mu$-classes. Relations between the values of the $\mu$-classes of $(\mathscr{F}, \mu)$ and the global geometry of $\mathscr{F}$ are investigated in this section. Examples to illustrate these properties are given in $\S 4$.

Let $(\mathscr{F}, \mu)$ be a measured foliation on $M$. Recall that a set $X \subseteq M$ is saturated if for each leaf $L$ of $\mathscr{F}$ with $L \cap X \neq \varnothing$ we have $L \subseteq X$. For a $\mu$-measurable saturated subset $X$ of $M$, denote by $\alpha_{X}: M \rightarrow \mathbf{R}$ the characteristic function which is equal to 1 on $X$ and zero otherwise. Then $\mu \cdot \alpha_{X}$ is an invariant measure on $M$ and we can define $\mu$-classes for $\left(\mathcal{F}, \mu \cdot \alpha_{X}\right)$. Given a countable decomposition of $M$ into saturated, $\mu$-measurable sets $\left\{X_{i}\right\}$ with $M=\dot{U} X_{i}$, we set $\mu_{i}=\mu \cdot \alpha_{X_{i}}$ and so $\mu=\Sigma \mu_{i}$. For $M$ a closed manifold, we then have $\chi_{\mu}=\Sigma \chi_{\mu_{i}}$ showing the $\mu$-classes can be decomposed into the contributions from invariant subsets of $M$.

The $\mu$-classes are measured-concordance invariants. If $\left(\mathscr{F}_{0}, \mu_{0}\right)$ and $\left(\mathscr{F}_{1}, \mu_{1}\right)$ are measured foliations on $M$ and the characteristic maps $\chi_{\mu_{0}} \neq \chi_{\mu_{1}}$, then there does not exist a measured foliation $(\mathscr{F}, \mu)$ on $M \times[0,1]$ which restricts to these two foliations on the ends. For example, when each $\mu_{i}$ arises from counting the intersections of a transversal with a compact leaf $L_{i}$ of $\mathscr{F}_{i}$, this implies there is no codimension $q$ foliation $\mathscr{F}$ on $M \times[0,1]$ with a compact leaf $L$ so that $(\mathscr{F}, L)$ restricts to $\left(\mathscr{F}_{i}, L_{i}\right)$ on $M \times\{i\}$. However, there may well be a foliation $\mathscr{F}$ restricting to the given foliations $\mathscr{F}_{i}$. In this situation, the $\mu$-classes are obstructions to the existence of compact leaves which satisfy a boundary condition.

The support of a transverse invariant measure $\mu$ is always a saturated set. When the support consists entirely of compact leaves, then the $\mu$-classes are precisely the leaf classes of $\mathcal{F}$ weighted according to $\mu$. Let $M$ be compact without boundary. We first consider the case when support $\mu$ consists of a single compaci leaf $L$. Up to a scalar multiple, $\mu$ is the counting measure with respect to $L$, and without loss of generality we can assume $\mu=\mu_{L}$. Assume that $Q, \mathcal{F}$ and $M$ are oriented, so for 
$z \in H^{l}(M)$, respectively $w \in H^{l}(L)$, there is a well-defined Poincaré dual $z^{\prime} \in$ $H_{m-l}(M)$, respectively $w^{\prime} \in H_{n-l}(L)$, where $n=m-q=\operatorname{dimension} L$. As above, $j: L \rightarrow M$ is the inclusion and $\chi_{L}: H^{*}\left(g l_{q}, O_{q}\right) \rightarrow H^{*}(L)$ is the leaf class map.

Proposition 3.1. (a) There is a commutative diagram:

$$
\begin{array}{ccccc} 
& \chi_{\mu_{L}} \nearrow & H^{l+q}(M) \underset{\text { P.D. }}{\cong} & H_{n-l}(M) \\
H^{\prime}\left(g l_{q}, O_{q}\right) & & & & \uparrow j_{*} \\
& & & & \\
& \chi_{L} \searrow & H^{\prime}(L) & \stackrel{\text { P.D. }}{\cong} & H_{n-l}(L)
\end{array}
$$

(b) If $Q$ has a framing $s$, then there is a corresponding relationship between the maps $\chi^{s}$ and $\chi_{L}^{s}$.

Proof. The map $\chi_{\mu}$ is defined via the map into the currents, $\tilde{\chi}_{\mu}: H^{l}\left(g l_{q}, O_{q}\right) \rightarrow$ $\operatorname{Hom}\left(H^{n-l}(M), \mathbf{R}\right) \cong H_{n-l}(M)$. For a closed form $\phi \in \mathbb{Q}^{n-l}(M)$ and $y \in$ $H^{l}\left(g l_{q}, O_{q}\right)$, formula (1.1) reduces to $\tilde{\chi}_{\mu}(y)[\phi]=\int_{L} \Delta(y) \wedge \phi$. This is clearly the same as restricting $[\phi]$ to $H^{n-l}(L)$ and pairing with the leaf class $\chi_{L}(y)$, which in turn is the same as evaluating $[\phi]$ on $j_{*}\left[\chi_{L}(y)^{\prime}\right]$. The proof of (b) for $\chi_{\mu}^{s}$ is identical.

When the support of $\mu$ is the union of compact leaves, we have a similar but more delicate relationship between $\chi_{\mu}$ and the leaf classes. Let $T=\operatorname{support} \mu / \mathscr{F}$ be the transversal space of the support $\mu$. This is a possibly singular space, but it still makes sense to integrate with respect to $\mu$ over $T[36, \S 6]$. For each $x \in T$, let $L_{x}$ be the corresponding leaf in support $\mu$ with leaf classes $\chi_{L_{x}}: H^{*}\left(g l_{q}, O_{q}\right) \rightarrow H^{*}\left(L_{x}\right)$. Given $y \in H^{l}\left(g l_{q}, O_{q}\right)$ and $[\phi] \in H^{n-l}(M)$ we obtain a real number $\int_{L_{x}} \chi_{L_{x}}(y) \wedge \phi$.

Proposition 3.2. With notation as above,

$$
\tilde{\chi}_{\mu}(y)[\phi]=\int_{T}\left\{\int_{L_{x}} \chi_{L_{x}}(y) \wedge \phi\right\} \cdot \mu .
$$

Further, a corresponding formula holds for $\tilde{\chi}_{\mu}^{s}$.

Proof. This follows directly from our definitions and [36, Proposition 6.1].

COROLlARY 3.3. Let $(\mathscr{F}, \mu)$ be a measured foliation on a closed manifold and assume support $\mu$ is the union of compact leaves. Then $\chi_{\mu}$ depends only on the $C^{1}$-germ of $\mathcal{F}$ about support $\mu$. Given a $y \in H^{l}\left(g l_{q}, O_{q}\right)$ with $\chi_{L}(y)=0$ for all leaves $L$ in support $\mu$, then $\chi_{\mu}(y)=0$ also.

Proof. By 3.2, $\tilde{\chi}_{\mu}$ and hence $\chi_{\mu}$ depends only on $\mu$ and the leaf classes $\chi_{L}(y)$ for $L \subseteq$ support $\mu$, and the leaf classes of $L$ depend only on the $C^{1}$-germ of $\mathscr{F}$ about $L$. The vanishing condition follows from 3.2 directly.

An important question is whether Proposition 3.2 has an extension to measures whose supports contain noncompact leaves. We mention one application of 3.2. It is shown in [21] that if a leaf $L$ of a foliation $\mathscr{F}$ has a nontrivial leaf class of degree greater than one, then the linear holonomy group of the leaf must have exponential 
growth, and there is a leaf of $\mathscr{F}$ near $L$ with exponential growth. Combined with 3.3 this yields

Proposition 3.4. Let $(\mathscr{F}, \mu)$ be a measured foliation on a compact manifold without boundary and assume the support of $\mu$ consists entirely of compact leaves. If $\mathcal{F}$ has subexponential growth in an open neighborhood of support $\mu$, then $\chi_{\mu}(y)=0$ for all $y \in H^{l}\left(g l_{q}, O_{q}\right), l>1$.

A theorem of Hirsch and Thurston [16] states that the Euler class of a flat bundle vanishes if there is a transverse invariant measure for the foliation defined by the flat structure. For the germ of a foliation about a union of compact leaves, Proposition 3.4 can be viewed as a generalization of this result. The extra hypothesis on the growth of the leaves of $\mathscr{F}$ forces all of the differential invariants of the normal flat bundle to vanish in degrees $>1$.

It is not possible to extend 3.4 to the invariants from $H^{*}\left(g l_{q}\right)$. For a measured foliation $(\mathscr{F}, \mu)$ with framing $s$, the value of $\chi_{\mu}^{s}$ on the cokernel of $H^{*}\left(g l_{q}, O_{q}\right) \rightarrow$ $H^{*}\left(g l_{q}\right)$ depends upon the framing $s$. For example, let $\mathscr{F}$ be the foliation defined by the standard fibration $\pi: S O_{q+1} \rightarrow S^{q}$. A volume form $\tilde{\omega}$ on $S^{q}$ lifts to a transverse volume form $\omega=\pi^{*} \tilde{\omega}$. The normal bundle $Q \cong \pi^{*} T S^{q}$ has a canonical framing $s$, so we obtain a framed, measured foliation $(\mathscr{F}, \mu, s)$. For $q \geqslant 3$ odd, the class $[\omega] \in$ $H^{q}\left(S O_{q+1}\right)$ is nonzero. The Chern-Weil definition of $\Delta^{s}$ shows each $\Delta^{s}\left(y_{2 i}\right)$ restricts to a generator of $H^{*}\left(S O_{q}\right)$. It is then easy to see that $\chi_{\mu}: H^{*}\left(g l_{q}\right) \rightarrow H^{*+q}\left(S O_{q+1}\right)$ is injective on the exterior algebra generated by the monomials $\left\{y_{2 i}\right\}$. But all leaves of $\mathscr{F}$ are compact ( $\cong S O_{q}$ ) and have no holonomy. The key ingredient in this example is that the restriction of the framing $s$ to each leaf $L=S O_{q}$ is homotopic to the identity.

The above example illustrates a property of foliations which has been called the permanence principle $[23,19,33]$. We state a general consequence of this for the $\mu$-classes. Let $(\mathscr{F}, \mu)$ be a measured foliation on $M$ with framing $s$ of the normal bundle $Q$. Let $N=M \times S O_{q}$ denote the product and $\left(\mathcal{F}^{\prime}, \mu^{\prime}\right)$ the pull-back measured foliation on $N$ via the projection $p: N \rightarrow M$. Define a framing $s^{\prime}$ of $Q^{\prime}=p^{*} Q$ by setting, for $(m, g) \in N, s^{\prime}(m, g)=s(m) \cdot g$. The framing $s^{\prime}$ of $Q^{\prime}$ is the framing $s \circ p$ twisted by the action of $G$.

THEOREM 3.5. Let $(\mathscr{F}, \mu, s)$ on $M$ and $\left(F^{\prime}, \mu^{\prime}, s^{\prime}\right)$ on $N=M \times S O_{q}$ be as above. Assume $\chi_{\mu}: H^{*}\left(g l_{q}, O_{q}\right) \rightarrow H^{*+q}(M)$ is injective on a subspace $V \subseteq H^{*}\left(g l_{q}, O_{q}\right)$. Then $\chi_{\mu^{\prime}}^{s^{\prime}}: H^{*}\left(g l_{q}\right) \rightarrow H^{*+q}(N)$ is injective on the extended subspace $\bar{V}=V * H^{*}\left(S O_{q}\right)$

Here, $V * H^{*}\left(S O_{q}\right)$ denotes the subspace of $H^{*}\left(g l_{q}\right)$ spanned by the elements in $\Lambda\left(y_{1}, y_{2}, \ldots, y_{q}\right)$ of the form $\left\{z \wedge y_{2 i_{1}} \wedge \cdots \wedge y_{2 i_{r}} \mid z \in V ; 1 \leqslant i_{1}<\cdots<i_{r}<q / 2\right\}$.

The proof of 3.5 proceeds exactly as that of [19, Theorem 3.5].

When support $\mu$ is a single compact leaf $L$, Theorem 3.5 has an appealing interpretation. By 3.1, $\tilde{\chi}_{\mu}^{s}$ is the composition of $\chi_{L}^{s}$ with Poincare duality and then the inclusion $H_{*}(L) \rightarrow H_{*}(M)$. Thus, $\tilde{\chi}_{\mu}^{s}$ is determined by the map $\chi_{L}^{s}$ which is determined, in turn, by the framed linear holonomy $B(d h, s): L \rightarrow B \overline{G l} \mathbf{R}$. Note 
there is a fibration $S O_{q} \simeq \Omega B G l_{q}^{+} \rightarrow B \overline{G l} \mathbf{R} \rightarrow B G l_{q}^{\delta} \mathbf{R}^{+}$and $H^{*}(B \overline{G l} \mathbf{R}) \cong$ $H^{*}\left(B G l_{q}^{\delta} \mathbf{R}^{+}\right) \otimes H^{*}\left(S O_{q}\right)$. These remarks are also true for the leaf $L \times S O_{q} \subseteq M \times$ $S O_{q}=N$ with framing $s^{\prime}$, and this yields a commutative diagram:

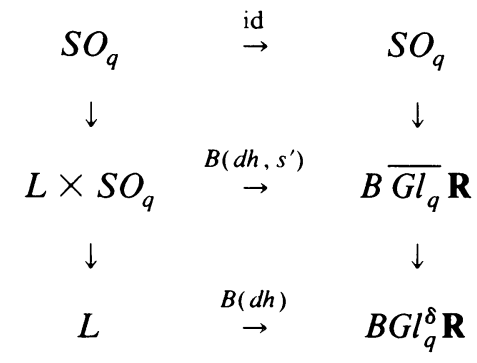

Thus, $B\left(d h, s^{\prime}\right)^{*}=B(d h)^{*} \otimes \mathrm{id}^{*}$ which implies 3.5 in this case.

4. Measured foliations with nonvanishing $\mu$-classes. A one-dimensional foliation on a compact riemannian manifold $M$ always has a transverse invariant measure. Let $v$ be a vector field on $M$ defining the foliation. If $v$ has a closed orbit $L$, then we can take the counting measure $\mu_{L}$. In general, given a noncompact orbit $\mathbf{R} \hookrightarrow M$ we use the metric to construct an asymptotic 1-cycle to which $\mu$ is dual [29]. For the measure $\mu_{L}$ we can evaluate the $\mu$-classes:

LEMMA 4.1. Let $v$ be a vector field on a compact manifold $M^{m}$ with a closed orbit $L$, and $\mu$ the counting measure for L. Let $A \in G l_{m-1} \mathbf{R}$ be the generator of the linear holonomy of $L$. Then $\chi_{\mu}\left(y_{1}\right) \in H^{m}(M)$ satisfies

$$
\left\langle\chi_{\mu}\left(y_{1}\right),[M]\right\rangle=-\frac{1}{2 \pi} \log |\operatorname{det} A| .
$$

Proof. By 3.1 we have $\left\langle\chi_{\mu}\left(y_{1}\right),[M]\right\rangle=\left\langle\chi_{L}\left(y_{1}\right),[L]\right\rangle$ and it is a classical result due to Reeb (see p. 143 of [22]) that

$$
\left\langle\chi_{L}\left(y_{1}\right),[L]\right\rangle=\int_{L} \Delta\left(y_{1}\right)=-\frac{1}{2 \pi} \log |\operatorname{det}(\operatorname{dh}(1))|
$$

where $d h: \pi_{1}(L) \rightarrow G l_{m-1} \mathbf{R}$ is the linear holonomy and $1 \in \pi_{1}(L)$ is the generator.

EXAMPLE 4.2. Let $v$ be a nonvanishing vector field on $T^{2}$ with a closed orbit $L$, and suppose $L$ is an attractor so that $d h: \pi_{1}(L) \rightarrow G l_{1} \mathbf{R}$ has $\alpha=|d h(1)|<1$. Then $\left\langle\chi_{\mu}\left(y_{1}\right),\left[T^{2}\right]\right\rangle=1 / 2 \pi \cdot \log (1 / \alpha)$ is positive.

Note that if $L_{1}, \ldots, L_{n}$ are closed orbits of $v$ on $M$ and $\mu=\sum_{i=1}^{n} \lambda_{i} \mu_{L_{i}}$, then we can extend 4.1 to

$$
\left\langle\chi_{\mu}\left(y_{1}\right),[M]\right\rangle=-\frac{1}{2 \pi} \sum_{i=1}^{n} \lambda_{i} \log \left|\operatorname{det} A_{i}\right|,
$$

where $A_{i} \in G l_{m-1} \mathbf{R}$ is the generator of the linear holonomy of $L_{i}$. Choose a vector field on $T^{2}$ with 3 closed orbits $L_{1}, L_{2}, L_{3}$ all in the same homology class and with $A_{1}=A_{3}=(1 / 2), A_{2}=(2)$. Let $\mu=\mu_{L_{1}}-2 \mu_{L_{2}}+\mu_{L_{3}}$. Then $\chi_{\mu}(1)=[\mu] \in H^{1}\left(T^{2}\right)$ is zero but $\left\langle\chi_{\mu}\left(y_{1}\right),\left[T^{2}\right]\right\rangle=(2 / \pi) \log 2 \neq 0$. This shows the two classes $\chi_{\mu}(1)$ and $\chi_{\mu}\left(y_{1}\right)$ can be independent for a given foliation. 
EXAmple 4.4. A less artificial example showing the independence of $\chi_{\mu}(1)$ and $\chi_{\mu}\left(y_{1}\right)$ is easily obtained. Let $v$ be a vector field on $S^{3}$ with a closed orbit $L$ and assume the linear holonomy of $L$ is generated by $A \in G l_{2} \mathbf{R}$. If we construct $v$ so that $A$ has eigenvalues $0<\lambda_{1} \leqslant \lambda_{2}<1$, hence $L$ is an attractor, then

$$
\left\langle\chi_{\mu}\left(y_{1}\right),\left[S^{3}\right]\right\rangle=-\frac{1}{2 \pi} \log \lambda_{1} \lambda_{2}>0 .
$$

Thus $\chi_{\mu}\left(y_{1}\right) \neq 0$ but $\chi_{\mu}(1) \in H^{2}\left(S^{3}\right)$ always vanishes.

The leaf classes of a foliation measure the dynamical behavior of $\mathscr{F}$ in a neighborhood of a leaf, where exponential growth of the leaves corresponds to "mixing" in the orbit space and seems to be necessary for the leaf classes to be nontrivial. One point of the above example is that the $\mu$-classes provide nontrivial cohomology invariants of $(\mathscr{F}, \mu)$ by incorporating, with $\mu$, the geometric data inherent in the leaf classes. A similar phenomenon occurs with the secondary classes, where the Chern ring of the normal bundle can be trivial, but taking a product with the leaf classes produces interesting global invariants.

We next discuss a general process for constructing measured foliations about which the $\mu$-classes yield important information. The following concepts are well known, and we simply adapt them to our setting. A full treatment is found in Bott [5], for example.

Let $X$ be a connected manifold of dimension $q$. Diff ${ }^{c} X$ will denote the group of compactly supported diffeomorphisms: for $\phi \in \operatorname{Diff}^{c} X$, there is a compact set $K \subseteq X$ so that $\phi$ is the identity outside of $K$. We next are given a smooth manifold $B$ with fundamental group $\Gamma=\pi_{1}(B)$ and a representation $\Gamma \rightarrow \operatorname{Diff}^{c} X$. The universal cover $\tilde{B}$ of $B$ has a natural left $\Gamma$-action, so $\Gamma$ acts on the product $\tilde{B} \times \Gamma$ and we form the quotient manifold $M=\tilde{B} \times{ }_{\Gamma} X$. The horizontal foliation on $\tilde{B} \times X$ with leaves $\tilde{B} \times p t$ descends to a foliation $\mathscr{F}$ on $M$, transverse to the fibers of the projection $\pi$ : $M \rightarrow B$. If there is a measure $\tilde{\mu}$ on $X$ invariant under the $\Gamma$-action, then $\mathscr{F}$ inherits a transverse invariant measure $\mu$ and $(\mathscr{F}, \mu)$ is a measured foliation of codimension $q$ on $M$.

If we assume that $\tilde{\mu}$ is absolutely continuous, then $\mu$ is represented by a $q$-form $\omega$ with locally-integrable coefficients and there is a well-defined characteristic map $\chi_{\mu}$ : $H^{l}\left(g l_{q}, O_{q}\right) \rightarrow H_{c}^{l+q}(M)$ for $l>0$. Here, $H_{c}^{*}(M)$ denotes the cohomology of the cochains on $M$ which have compact support on each fiber of $M \rightarrow B$. To obtain $\chi_{\mu}$ we use a Bott connection on $M$ which is horizontal outside of a compact set in each fiber; such exists because $\Gamma$ acts through $\operatorname{Diff}^{c} X$. The interest in the group $H_{c}^{*}(M)$ is due to the existence of a map $\pi_{*}: H_{c}^{l+q}(M) \rightarrow H^{l}(B)$, defined by integration over the fiber [4, p. 15]. The composition $\pi_{*} \circ \chi_{\mu}: H^{*}\left(g l_{q}, O_{q}\right) \rightarrow H^{*}(B)$ defines invariants for the representation $\Gamma \rightarrow \operatorname{Diff}^{c} X$, and is functorial. We present an example below where these invariants have been extensively studied. In general, little is known about them.

For an absolutely continuous measure $\tilde{\mu}$ on $X^{q}$, let $\operatorname{Diff}_{\tilde{\mu}}^{c} X$ denote the discrete group of compactly supported diffeomorphisms which leave $\tilde{\mu}$ invariant. The constructions above yield the following. 
Proposition 4.5. There is a well-defined characteristic homomorphism

$$
\hat{\chi}_{\mu}=\pi_{*} \circ \chi_{\mu}: H^{*}\left(g l_{q}, O_{q}\right) \rightarrow H^{*}\left(B \operatorname{Diff}_{\tilde{\mu}}^{c} X\right) .
$$

The class $\chi_{\mu}\left(y_{\mathrm{i}}\right)$ always vanishes if $\mu$ is absolutely continuous, since the Weil operator $\chi\left(y_{1}\right)$ vanishes on the support of an absolutely continuous measure [15]. The first nontrivial invariant is thus $\hat{\chi}_{\mu}\left(y_{3}\right)$ in degree 5, defined for $q \geqslant 3$. A natural question is whether there exists an absolutely continuous measure on a closed 3-manifold $X$ and a subgroup $\Gamma \subseteq \operatorname{Diff}_{\tilde{\mu}} X$ for which $\hat{\chi}_{\mu}\left(y_{3}\right) \in H^{5}(\Gamma)$ is nonzero.

EXAMPLE 4.6. For higher codimensions, the following type of example was suggested to us by W. Thurston. We take $X=T^{q}$ the $q$-torus, and the measure $\tilde{\mu}$ is given by $\omega=d x_{1} \wedge \cdots \wedge d x_{q}$, the natural euclidean volume form. Then $S L_{q} Z \subseteq$ $\operatorname{Diff}_{\omega} T^{q}$, and $\hat{\chi}_{\omega}: H^{*}\left(g l_{q}, O_{q}\right) \rightarrow H^{*}\left(S L_{q} Z\right)$ can be identified with the composition $H^{*}\left(g l_{q}, O_{q}\right) \cong H_{c}^{*}\left(G l_{q}^{\delta} \mathbf{R}\right) \rightarrow H^{*}\left(B G l_{q}^{\delta} \mathbf{R}\right) \rightarrow H^{*}\left(S l_{q} \mathbf{Z}\right)$, where $H_{c}\left(G l_{q}^{\delta} \mathbf{R}\right)$ is the continuous cohomology of $G l_{q}^{\delta} \mathbf{R}$. Consequently, $\hat{\chi}_{\omega}\left(y_{3}\right)$ is nontrivial for $q \geqslant 25$ by Borel [3]. This example is used in part II, so we develop it in more detail.

Form the homogeneous space $\tilde{B}=S L_{q} \mathbf{R} / S O_{q}$ which is contractible. By Borel there is a subgroup $\Gamma \subseteq S L_{q} Z$ of finite index such that $\Gamma \backslash \tilde{B}=B$ is a smooth manifold. For each real $\lambda \neq 0$ the form $\tilde{\omega}_{\lambda}=\lambda \cdot d x_{1} \wedge \cdots \wedge d x_{q}$ is nonvanishing on $T^{q}$, and the natural $\Gamma$-action on $T^{q}$ preserves $\tilde{\omega}_{\lambda}$. We thus obtain a codimension $q$ measured foliation $\left(\mathscr{F}, \omega_{\lambda}\right)$ on $M=\tilde{B} \times{ }_{\Gamma} T^{q}$ which is transverse to the fibers of $\pi$ : $M \rightarrow B$. We calculate the characteristic map $\chi_{\omega_{\lambda}}: H^{*}\left(g l_{q}, O_{q}\right) \rightarrow H^{*+q}(M)$.

Associated to the inclusion $\Gamma \subseteq S L_{q} \mathbf{R}$ is a flat bundle $p^{\prime}: E=\tilde{B} \times{ }_{\Gamma} \mathbf{R}^{q} \rightarrow B$. The normal bundle $Q$ to the foliation $\mathcal{F}$ is the pull-back:

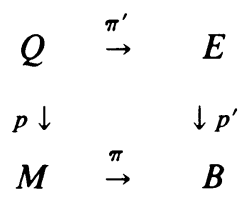

There is a given flat connection $\theta^{\prime}$ on $E \rightarrow B$, and it is easy to check that this pulls back under $\pi$ to a Bott connection $\theta$ for $\mathscr{F}$ on $Q \rightarrow M$. Hence $\left(\mathscr{F}, \omega_{\lambda}\right)$ admits a globally flat Bott connection $\theta$ which we use to construct $\Delta: W O_{q} \rightarrow \mathbb{Q}(M)$. The Chern forms $\Delta\left(c_{i}\right)$ vanish identically, so each $\Delta\left(y_{i}\right) \in \mathbb{Q}(M)$ is a closed form. Further, for

$$
\chi_{E}: H^{*}\left(g l_{q}, O_{q}\right) \rightarrow H^{*}(B),
$$

the characteristic map of the flat bundle $E \rightarrow B$, the functoriality of the Chern-Weil homomorphism implies $\pi^{*} \circ \chi_{E}\left(y_{i}\right)=\left[\Delta\left(y_{i}\right)\right] \in H^{2 i-1}(M)$. Recall that $\pi_{*}$ : $H^{*+q}(M) \rightarrow H^{*}(B)$ is the integration over the fiber map, and $\lambda \cdot \chi_{E}$ denotes $\chi_{E}$ scaled by a factor of $\lambda$.

LEMMA 4.7. The characteristic map $\chi_{\omega_{\lambda}}$ for $\left(\mathscr{F}, \omega_{\lambda}\right)$ factors:

$$
\begin{array}{ccc}
H^{*}\left(g l_{q}, O_{q}\right) & \stackrel{\lambda \cdot \chi_{E}}{\rightarrow} & H^{*}(B) \\
\chi_{\omega_{\lambda} \searrow} & & \nearrow \pi_{*} \\
& H^{*+q}(M) &
\end{array}
$$


Proof. Let $y \in H^{*}\left(g l_{q}, O_{q}\right)$. Then

$$
\begin{aligned}
\pi_{*} \circ \chi_{\omega_{\lambda}}(y) & =\pi_{*}\left(\left[\Delta(y) \wedge \omega_{\lambda}\right]\right)=\pi_{*}\left(\pi^{*}\left(\chi_{E}(y)\right) \cup\left[\omega_{\lambda}\right]\right) \\
& =\chi_{E}(y) \cup \pi_{*}\left(\left[\omega_{\lambda}\right]\right)=\lambda \cdot \chi_{E}(y),
\end{aligned}
$$

the last step because $\pi_{*}\left(\left[\omega_{\lambda}\right]\right)=\int_{T^{q}} \lambda d x_{1} \wedge \cdots \wedge ; d x_{q}=\lambda$.

Lemma 4.7 reduces the calculation of $\chi_{\omega_{\lambda}}$ to the problem of determining the characteristic classes for the flat bundle $E$ associated to $\Gamma \subseteq S L_{q} \mathbf{R}$. This is a very difficult problem, but it has been partially solved by Borel [3] who shows that $\chi_{E}$ : $H^{l}\left(s l_{q}, O_{q}\right) \rightarrow H^{l}(B) \cong H^{l}(\Gamma)$ is injective for $l<[(q-1) / 4]$.

The lowest codimension for which these examples have significance is $q=25$; the above arguments show $\chi_{\omega_{\lambda}}\left(y_{3}\right) \in H^{30}\left(\tilde{B} \times_{\Gamma} T^{25}\right)$ is nonvanishing and varies with $\lambda$.

$$
\text { II. } S L_{q} \text {-FOLIATIONS }
$$

5. The $\mu$-classes of $S L_{q}$-foliations. An $S L_{q}$-foliation on $M$ is a measured foliation $(\mathscr{F}, \mu)$ where $\mu$ is represented by a smooth nonvanishing $q$-form $\omega$. An $S L_{q}$-foliation admits an atlas of foliation charts $\left\{\phi_{\alpha}: U_{\alpha} \rightarrow \mathbf{R}^{q}\right\}$ for which

$$
\left.\omega\right|_{U_{\alpha}}=\phi_{\alpha}^{*}\left(d x_{1} \wedge \cdots \wedge d x_{q}\right)
$$

[10]. This yields the equivalent definition that an $S L_{q}$-foliation is one modeled on $\mathbf{R}^{q}$ whose transition functions preserve the volume form $d x_{1} \wedge \cdots \wedge d x_{q}$.

There are two standard ways to obtain examples of $S L_{q}$-foliations. Given a submersion $\pi: M \rightarrow X^{q}$ of smooth manifolds, the choice of a volume form $\tilde{\omega}$ on $X$ defines a closed $q$-form $\omega=\pi^{*} \tilde{\omega}$ which is transverse to the foliation $\mathcal{F}$ of $M$ by the fibers of $\pi$, yielding an $S L_{q}$-foliation $(\mathscr{F}, \omega)$. The other type of example is that of $\S 4$. Let $\tilde{\omega}$ be a volume form on $X^{q}$, and $\operatorname{Diff}_{\tilde{\omega}}^{c} X$ denotes the group of compactly supported diffeomorphisms which preserve $\tilde{\omega}$. Assume a representation $\Gamma \rightarrow \operatorname{Diff}_{\tilde{\omega}}^{c} X$ is given where $\Gamma=\pi_{1}(B)$ is the fundamental group of a manifold $B$. Then $\Gamma$ acts diagonally on the product $\tilde{B} \times X$, and the quotient manifold $M=\tilde{B} \times_{\Gamma} X$ has a natural codimension $q$ foliation with invariant volume form $\omega$ induced from $\tilde{\omega}$. The $\mu$-classes can be nontrivial for both of the above types of $S L_{q}$-foliations.

Let $B \Gamma_{S L_{q}}$ denote Haefliger's classifying space of $S L_{q}$-foliations [10]. The universal normal bundle is classified by $\nu: B \Gamma_{S L_{q}} \rightarrow B S L_{q}$, and $B \bar{\Gamma}_{S L_{q}}$ denotes the homotopy fiber of $\nu$. A basic property of $B \Gamma_{S L_{q}}$ is that an $S L_{q}$-foliation $(\mathscr{F}, \mu)$ on $M$ determines a unique homotopy class of maps $f: M \rightarrow B \Gamma_{S L_{q}}$. If the normal bundle $Q$ of $\mathscr{F}$ is trivial, then a choice of framing, $s$, determines a unique homotopy class of maps $f_{s}$ : $M \rightarrow B \bar{\Gamma}_{S L_{q}}$. The functoriality of the $\mu$-classes implies there are universal characteristic maps

$$
\chi_{\omega}: H^{*}\left(s l_{q}, O_{q}\right) \rightarrow H^{*+q}\left(B \Gamma_{S L_{q}}\right), \quad \chi_{\omega}^{s}: H^{*}\left(s l_{q}\right) \rightarrow H^{*+q}\left(B \bar{\Gamma}_{S L_{q}}\right) .
$$

We restrict the domains to the cohomology of $s l_{q}$ because an $S L_{q}$-foliation always admits a Bott connection for which the form $\Delta\left(y_{1}\right)$ vanishes identically, and thus $\chi_{\omega}\left(y_{I}\right)=0$ whenever $1 \in I$.

The classes in the image of $\chi_{\omega}$ and $\chi_{\omega}^{s}$ define elements in the differentiable cohomology of $\Gamma_{S L_{q}}$ and $\bar{\Gamma}_{S L_{q}}$, and therefore by [11] they come from the Gelfand-Fuks 
cohomology of the divergence-free vector fields on $\mathbf{R}^{q}$, as was pointed out to us by Haefliger. A further discussion on this is in the appendix to McDuff [27].

At present, the following is known about the spaces $B \Gamma_{S L_{q}}$ and $B \bar{\Gamma}_{S L_{q}}$. First, $B \bar{\Gamma}_{S L_{q}}$ is $(q-1)$-connected (Haefliger, [10]). McDuff has shown in [24] that $\pi_{q}\left(B \bar{\Gamma}_{S L_{q}}\right)=\mathbf{R}$, with the volume class $[\omega] \in H^{q}\left(B \bar{\Gamma}_{S L_{q}}\right)$ the complete invariant. Using a generalization of the Mather-Thurston theorem, McDuff also shows $\pi_{q+1}\left(B \bar{\Gamma}_{S L_{q}}\right)=0$ for $q>2$. The group $\pi_{3}\left(B \bar{\Gamma}_{S L_{2}}\right)=\mathbf{R}$ by Banyaga [1]. For degrees $n>2 q$, note that the examples of Heitsch $[13, \S 5]$ can be chosen to have the vector field $X_{\lambda}$ divergence-free, so they provide a family of examples of $S L_{q}$-foliations with nonvanishing secondary classes. Consequently, we see $H^{n}\left(B \Gamma_{S L_{q}}\right)$ and $H^{n}\left(B \bar{\Gamma}_{S L_{q}}\right)$ are nonvanishing for $n=2 q+1$ and many values of $n>2 q$. The variability of the secondary classes in the Heitsch examples implies $H_{2 q+1}\left(B \bar{\Gamma}_{S L_{q}} ; Z\right)$ maps onto a real vector space. Because the volume class $[\omega]$ is zero in the Heitsch examples, one can show this implies $\pi_{2 q+1}\left(B \bar{\Gamma}_{S L_{q}}\right)$ maps onto a real vector space also. There is a gap in this information about the homotopy groups $\pi_{*}\left(B \bar{\Gamma}_{S L_{q}}\right)$; this gap in degrees between $q+1$ and $2 q+1$ is filled by the $\mu$-classes:

THEOREM 5.1. The characteristic map $\chi_{\omega}: H^{l}\left(s l_{q}, O_{q}\right) \rightarrow H^{l+q}\left(B \Gamma_{S L_{q}}\right)$ is injective for $l<[(q-1) / 4]$, and the classes in the image of $\chi_{\omega}$ are independently variable.

THEOREM 5.2. The characteristic map $\chi_{\omega}^{s}: H^{*}\left(s l_{q}\right) \rightarrow H^{*+q}\left(B \bar{\Gamma}_{S L_{q}}\right)$ is injective on the subspace spanned by the set $V_{q}=\left\{y_{I} \in H^{*}\left(s l_{q}\right) \mid I=\left(i_{1}, \ldots, i_{r}\right)\right.$ with $2 \leqslant i_{1}<$ $\cdots<i_{r}<2[(q+1) / 2]$ and $i_{l}$ odd implies $\left.i_{l} \leqslant[(q-1) / 8]\right\}$, and the set $\chi_{\omega}^{s}\left(V_{q}\right)$ consists of independently variable classes.

The following corollary of 5.2 can be taken as the definition of a set being independently variable.

COROLlaRY 5.3. Evaluation of the set $\chi_{\omega}^{s}\left(V_{q}\right)$ defines group epimorphisms

$$
H_{n+q}\left(B \bar{\Gamma}_{S L_{q}} ; Z\right) \rightarrow \operatorname{Span}_{\mathbf{R}} V_{q}(n)
$$

where $V_{q}(n)$ denotes the elements of $V_{q}$ of degree $n$.

The definition of the set $V_{q}$ is somewhat complicated; for small values of $q$ we give $V_{q}$ explicitly (recall that $y_{i}$ has degree $2 i-1$ and 1 has degree 0 ):

$$
V_{1}=V_{2}=\{1\}, \quad V_{3}=V_{4}=\Lambda_{z}\left\{1, y_{2}\right\}, \quad V_{5}=V_{6}=\Lambda_{Z}\left\{1, y_{2}, y_{4}\right\}
$$

and so forth up to

$$
V_{23}=V_{24}=\Lambda_{Z}\left\{1, y_{2}, y_{4}, \ldots, y_{22}\right\} \text {. }
$$

Then, we have up to codimension $q=42$ :

$$
\begin{aligned}
& V_{25}=V_{26}=\Lambda_{Z}\left\{1, y_{2}, \ldots, y_{24}\right\} \times\left\{1, y_{3}\right\}, \\
& V_{39}=V_{40}=\Lambda_{Z}\left\{1, y_{2}, \ldots, y_{38}\right\} \times\left\{1, y_{3}\right\}, \\
& V_{41}=V_{42}=\Lambda_{Z}\left\{1, y_{2}, \ldots, y_{40}\right\} \times\left\{1, y_{3}, y_{5}\right\} .
\end{aligned}
$$

The second set in these products is a basis for $H^{*}\left(s l_{q}, O_{q}\right)$ in degrees less than $[(q-1) / 4]$. 
Proof of 5.1. To show the universal map $\chi_{\omega}$ is injective on a set, it suffices to exhibit an $S L_{q}$-foliation for which the set is injective. In particular, in Example 4.6 we produced an $S L_{q}$-foliation $\left(\mathscr{F}, \omega_{\lambda}\right)$ on $M=S L_{q} / S O_{q} \times_{\Gamma} T^{q}$ for which $\chi_{\omega_{\lambda}}$ : $H^{n}\left(s l_{q}, O_{q}\right) \rightarrow H^{n+q}(M)$ is injective when $n<[(q-1) / 4]$, showing the first claim of 5.1. We next establish the independent variation; it suffices to check this for homogeneous cocycles. Choose a basis $\left\{z_{1}, \ldots, z_{a}\right\}$ of $H^{n}\left(s l_{q}, O_{q}\right)$ where $n<$ $[(q-1) / 4]$. Let $f_{\lambda}: M \rightarrow B \Gamma_{S L_{q}}$ be the classifying map of $\left(\mathscr{F}, \omega_{\lambda}\right)$. For $\lambda=1$, choose cycles $\left\{c_{1}, \ldots, c_{a}\right\} \subseteq H_{n+q}(M ; Z)$ so that $A_{i j}=\chi_{\omega}\left(z_{i}\right)\left(f_{1 *} c_{j}\right)=\chi_{\omega_{1}}\left(z_{i}\right)\left(c_{j}\right)$ is a nonsingular matrix. This is possible because $\chi_{\omega_{1}}$ is injective on the span of the set $\left\{z_{1}, \ldots, z_{a}\right\}$. Given an $a$-tuple of nonzero real numbers, $\bar{\lambda}=\left(\lambda_{1}, \ldots, \lambda_{a}\right)$, for the universal maps $f_{\lambda}$, we have $\chi_{\omega}\left(z_{i}\right)\left(\sum_{j=1}^{a} f_{\lambda_{j}} * z_{j}\right)=\sum_{j=1}^{a} A_{i j} \lambda_{j}$. Now, $A$ nonsingular implies these evaluations are onto an open set in $\mathbf{R}^{a}$. Therefore, evaluation of the set $\left\{\chi_{\omega}\left(z_{1}\right), \ldots, \chi_{\omega}\left(z_{a}\right)\right\} \subseteq H^{n+q}\left(B \bar{\Gamma}_{S L_{q}}\right)$ on cycles in $H_{n+q}\left(B \bar{\Gamma}_{S L_{q}} ; Z\right)$ defines a homomorphism onto $\mathbf{R}^{a}$.

Proof OF 5.2. We remarked at the end of $\S 3$ that a choice of a volume form $\tilde{\omega}$ on $S^{q}$ makes the fibration $S O_{q+1} \rightarrow S^{q}$ into an $S L_{q}$-foliation $(\mathscr{F}, \omega)$ on $S O_{q+1}$. Further, the normal bundle of $\mathscr{F}$ has a canonical framing for which $\chi_{\mu}^{s}: H^{*}\left(s l_{q}\right) \rightarrow H^{*}\left(S O_{q+1}\right)$ is injective on the coimage of $H^{*}\left(s l_{q}\right) \rightarrow H^{*}\left(s o_{q}\right)$ when $q$ is odd, and the classes are proportional to the value of $\int_{S^{4}} \tilde{\omega}$. Theorem 5.2 asserts that $\chi_{\omega}^{s}$ is injective on a larger set and for all $q$, so we must resort to a more rewarding example. The plan is to apply the permanence principle, Theorem 3.5, to the Example 4.6. The difficulty is that the normal bundle $Q$ of $\mathscr{F}$ on $M=S L_{q} / S O_{q} \times{ }_{\Gamma} T^{q}$ is not trivial, so we must lift the $S L_{q}$-foliation $\left(\mathscr{F}, \omega_{\lambda}\right)$ to the frame bundle of $Q$.

Let $p: P \rightarrow M$ denote the $S O_{q}$-bundle of orthonormal frames of $Q$ with respect to some metric on $Q$. Then $\left(\mathscr{F}, \omega_{\lambda}\right)$ lifts to an $S L_{q}$-foliation $\left(p^{*} \mathscr{F}, p^{*} \omega_{\lambda}\right)$ on $P$ and the functoriality of $\chi_{\mu}$ yields a commutative diagram:

$$
\begin{array}{ccc} 
& \chi_{p^{*} \mu} \nearrow & H^{*+q}(P) \\
& & \left.\uparrow l_{q}, O_{q}\right) \\
& \chi_{\mu} \searrow & H^{*+q}(M)
\end{array}
$$

If we show the injectivity of $\chi_{p^{* \mu}}$ in degrees less than $[(q-1) / 4]$, and since $p^{*} \mathscr{F}$ has a trivial normal bundle, we can then apply 3.5 to $\left(p^{*} \mathscr{F}, p^{*} \omega_{\lambda}\right)$ on $P$ to obtain the injectivity claim of 5.2. By the calculations of 4.6 , it thus suffices to show $p^{*}$ is injective on the image of $\chi_{\mu}$ in degrees less than $[(q-1) / 4]$.

For $q$ odd, $p^{*}$ is injective. Consider the Serre spectral sequence $E_{t}^{r, s}$ of the fibration $S O_{q} \rightarrow P \rightarrow{ }^{p} M$. The $E_{2}$ term is $H^{r}(M) \otimes H^{s}\left(S O_{q}\right)$ since the fundamental group $\Gamma$ of $M$ acts trivially on $H^{*}\left(S O_{q}\right)$. The transgression map defines the Pontrjagin and Euler classes of the normal bundle $Q$. But $q$ has odd dimension, and it was noted in 4.6 that $Q$ is a flat bundle, so all of its real primary classes are zero. Thus, the spectral sequence collapses, and $p^{*}: H^{*}(M) \rightarrow H^{*}(M) \otimes H^{*}\left(S O_{q}\right) \cong$ $H^{*}(P)$ is an inclusion.

For $q$ even, the Euler class of $Q$ is not zero, and we can only show $p^{*}$ is injective on special classes. Recall from 4.6 that $Q \rightarrow M$ is the pull-back of the flat bundle $E \rightarrow B$ via $\pi: M \rightarrow B$. Let $p_{0}: P_{0} \rightarrow B$ be the orthonormal bundle of frames of $E$ with 
respect to some metric. We can assume that $P$ is the pull-back in:

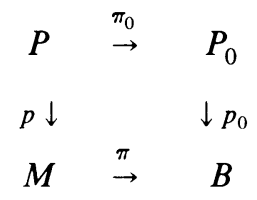

The Serre spectral sequence for the fibration $P_{0} \rightarrow B$ has $E_{2}^{r, s} \cong H^{r}(B) \otimes H^{s}\left(S O_{q}\right)$, and the transgression is zero in degrees less than $(q-1)$. (There is a class in $H^{q-1}\left(S O_{q}\right)$ which transgresses to the Euler class in $H^{q}(B)$.) This implies that for $r \leqslant q-1$, there is an inclusion $p_{0}^{*}: H^{r}(B) \rightarrow H^{r}\left(P_{0}\right)$.

Next, note the fibration $\pi_{0}: P \rightarrow P_{0}$ has fiber $T^{q}$ and the lift of the volume form evaluates nontrivially, $\left\langle p^{*}\left[\omega_{\lambda}\right],\left[T^{q}\right]\right\rangle=\lambda$. Therefore, $p^{*}\left[\omega_{\lambda}\right] \in H^{q}(P)$ is nonzero and for the fibration $P \rightarrow P_{0}$ we obtain an inclusion

$$
H^{*}\left(P_{0}\right) \rightarrow H^{*}\left(P_{0}\right) \otimes H^{q}\left(T^{q}\right) \stackrel{\cup}{\rightarrow} H^{*+q}(P) .
$$

Piecing these facts together and using Lemma 4.7, there is a commutative diagram:

$$
\begin{array}{cccc}
H^{s+q}(P) & \pi_{0}^{*} \cup p^{*}\left[\omega_{\lambda}\right] & H^{s}\left(P_{0}\right) \\
\begin{array}{c}
p^{*} \uparrow \\
H^{s+q}(M)
\end{array} & & \uparrow p_{0}^{*} \\
& & & H^{s}(B) \\
& \chi_{\mu_{\lambda}} \nwarrow & & \\
& & H^{s}\left(s l_{q}, S O_{q}\right) & \\
& & &
\end{array}
$$

The injectivity of $p^{*} \circ \chi_{\mu_{\lambda}}$ in degrees less than $[(q-1) / 4]$ now follows from (5.4) and the injectivity of $p_{0}^{*} \circ \chi_{E}$ in this range.

Next lift $\left(p^{*} \mathscr{F}, p^{*} \omega_{\lambda}\right)$ on $P$ to the product $P \times S O_{q}$ to get an $S L_{q}$-foliation $\left(\mathscr{F}^{\prime}, \omega_{\lambda}^{\prime}\right)$. The natural framing of $Q^{\prime}=p^{*} Q \rightarrow P$ is twisted via the action of the factor $S O_{q}$ to yield a new framing $s^{\prime}$ of $Q^{\prime} \rightarrow P \times S O_{q}$ as in the set-up for Theorem 3.5. We conclude that $\chi_{\mu_{\lambda}}^{s^{\prime}}: H^{*}\left(s l_{q}\right) \rightarrow H^{*+q}\left(P \times S O_{q}\right)$ maps $V_{q}$ to a linearly independent set, and the values of the image classes are directly proportional to $\lambda$. Finally, the independent variation of the set $\chi_{\omega}^{s}\left(V_{q}\right) \subseteq H^{*}\left(B \bar{\Gamma}_{S L_{q}}\right)$ is proved exactly as in 5.1 .

6. Homotopy groups of $B \bar{\Gamma}_{S L_{q}}$. The space $B \bar{\Gamma}_{S L_{q}}$ is $(q-1)$-connected, and the evaluation of the volume class on elements of $\pi_{q}\left(B \bar{\Gamma}_{S L_{q}}\right)$ defines a map $B \bar{\Gamma}_{S L_{q}} \rightarrow$ $K(\mathbf{R}, q)$ whose homotopy fiber is denoted $B \bar{\Gamma}_{S L_{q}}$. McDuff has shown $B \bar{\Gamma}_{S L_{q}}$ is $(q+1)$-connected if $q \geqslant 3$ [25]. As a consequence of 5.2, there are epimorphisms $\pi_{q+3}\left(B \bar{\Gamma}_{S L_{q}}\right) \rightarrow \mathbf{R}$ so $B \bar{\Gamma}_{S L_{q}}$ is not $(q+3)$-connected. This is one of the applications of Theorem 5.2 to the calculation of $\pi_{*}\left(B \bar{\Gamma}_{S L_{q}}\right)$. Fix $q \geqslant 3$. Recall that $V_{q} \subseteq H^{*}\left(s l_{q}\right)$ is the set defined in 5.2. Let $V_{q}(n)$ denote the subset of $V_{q}$ consisting of elements of degree $n$.

THEOREM 6.1. For $n<q$ the $\mu$-classes from the set $V_{q}(n)$ define an epimorphism of abelian groups

$$
\pi_{n+q}\left(B \bar{\Gamma}_{S L_{q}}\right) \rightarrow \operatorname{Span}_{\mathbf{R}} V_{q}(n)
$$


COROLlARY 6.2. For $n<q$ there is an inclusion of rational vector spaces

$$
\sigma: \operatorname{Span}_{\mathbf{R}} V_{q}(n) \rightarrow \pi_{n+q}\left(B \bar{\Gamma}_{S L_{q}}\right) \otimes \mathbf{Q} .
$$

Proof OF 6.2. Choose a Q-vector space section $\sigma$ of the epimorphism $\pi_{n+q}\left(B \bar{\Gamma}_{S L_{q}}\right)$ $\otimes \mathbf{Q} \rightarrow \operatorname{Span}_{\mathbf{R}} V_{q}(n)$ induced from the map in 6.1.

For the special case $q=3$ there are three basis elements of $H^{*}\left(s l_{3}\right)$, the classes $y_{2}$, $y_{3}, y_{2} y_{3}$. About the first we can show:

Proposition 6.3. Evaluation of $\chi_{\omega}^{s}\left(y_{2}\right) \in H^{6}\left(B \bar{\Gamma}_{S L_{3}}\right)$ defines an epimorphism of abelian groups $\pi_{6}\left(B \bar{\Gamma}_{S L_{3}}\right) \rightarrow \mathbf{R}$, with corresponding rational section $\sigma: \mathbf{R} \rightarrow \pi_{6}\left(B \bar{\Gamma}_{S L_{3}}\right)$ $\otimes \mathbf{Q}$.

We turn now to the proof of 6.1. Let $b_{n}$ be the number of elements in the set $V_{q}(n)$, and set $b=\max \left\{b_{n}\right\}$. For real $\lambda \neq 0$, let $P \times S O_{q}$ have the $S L_{q}$-foliation $\left(\mathscr{F}^{\prime}, \omega_{\lambda}^{\prime}\right)$ constructed in the proof of 5.2 , with normal framing $s^{\prime}$ and classifying map $f_{\lambda}^{s^{\prime}}: P \times S O_{q} \rightarrow B \bar{\Gamma}_{S L_{q}}$.

Next modify the space $P \times S O_{q}$ to eliminate the unnecessary structure in dimensions less than $q . P \times S O_{q}$ is a compact manifold and so has a finite $\mathrm{CW}$-complex structure. Attach a finite number of cells to $P \times S O_{q}$ of dimension $\leqslant q$ to obtain a $(q-1)$-connected space $W$. Each map $f_{\lambda}^{s^{\prime}}$ extends to a map $F_{\lambda}: W \rightarrow B \bar{\Gamma}_{S L_{q}}$ since the latter space is $(q-1)$-connected. There is a commutative diagram

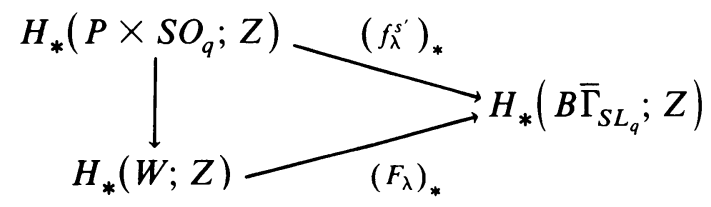

so the cycles in the image of $\left(f_{\lambda}^{s^{\prime}}\right)_{*}$ are also in the image of $\left(F_{\lambda}\right)_{*}$.

Let $Y$ be the one-point union of an uncountable number of copies of $W$, so $Y=\bigvee_{\lambda \in \mathbf{R}, \lambda \neq 0} W_{\lambda}$. Then set $X=\bigvee_{k=1}^{b} Y_{k}$, the one-point union of $b$-copies of $Y$. Construct $F: X \rightarrow B \bar{\Gamma}_{S L_{q}}$ where $F$ restricted to a factor $W_{\lambda, k} \subseteq Y_{k} \subseteq X$ is the map $F_{\lambda}$. The point of introducing $X$ and $F$ is that the proof of 5.1 and 5.2 shows the universal classes in the set $\chi_{\omega}^{s}\left(V_{q}\right) \subseteq H^{*}\left(B \bar{\Gamma}_{S L_{q}}\right)$ assume any prescribed values on the cycles in the image of $F_{*}: H_{*}(X ; Z) \rightarrow H_{*}\left(B \bar{\Gamma}_{S L_{q}} ; Z\right)$. The space $X$ is thus "universal" with respect to varying the $\mu$-classes in $\chi_{\omega}^{s}\left(V_{q}\right)$.

The high connectivity of $W$ enables us to analyze its rational homotopy groups using the rational Hurewicz theorem [2, Proposition 3.8]. To wit, there is an isomorphism $\mathcal{H}: \pi_{m}(W) \otimes \mathbf{Q} \rightarrow H_{m}(W ; \mathbf{Q})$ for $m<2 q-1$, and $\mathcal{H}$ is onto for $m=2 q-1$. Therefore, we can choose a set of homogeneous classes $\left\{g_{1}, \ldots, g_{r}\right\}$ in $\bigoplus_{m<2 q} \pi_{m}(W)$ which maps onto a basis $\left\{c_{1}, \ldots, c_{r}\right\}$ for $\bigoplus_{m<2 q} H_{m}(W ; \mathbf{Q})$. Each $g_{i}$ is represented by a map also denoted $g_{i}: S^{n_{i}} \rightarrow W$, so $c_{i}=\mathcal{H}\left(g_{i}\right)$ is an integral class in $H_{*}(W ; \mathbf{Q})$. For each inclusion of a factor $i_{\lambda, k}: W \rightarrow X$, the naturalicy of $\mathcal{H}$ yields a commutative diagram:

$$
\begin{array}{cccc}
\pi_{m}(W) & \rightarrow & H_{m}(W ; Z) \\
\left(i_{\lambda, k}\right)_{\#} \downarrow & & \downarrow\left(i_{\lambda, k}\right)_{*} \\
\pi_{m}(X) & \rightarrow & H_{m}(X ; Z)
\end{array}
$$


We have seen the cohomology classes $F^{*} \circ \chi_{\omega}^{s}\left(V_{q}\right) \subseteq H^{*}(X)$ assume any prescribed values when evaluated on cycles in the image of the maps $\left(i_{\lambda_{1}} \vee \cdots \vee i_{\lambda_{b}}\right)$ : $H_{*}(Y ; Z) \rightarrow H_{*}(X ; Z)$. For $m<2 q$, the cokernel of $\pi_{m}(Y) \rightarrow H_{m}(Y ; Z)$ is a finite abelian group. Consequently, for $n<q, F^{*} \circ \chi_{\omega}^{s}\left(V_{q}(n)\right)$ assumes any prescribed set of values when evaluated on spherical cycles in the image of $\left(i_{\lambda_{1}} \vee \cdots \vee i_{\lambda_{b}}\right)_{*} \circ \mathcal{H}$ : $\pi_{n+q}(Y) \rightarrow H_{n+q}(X ; Z)$ for the various choices of $\left(\lambda_{1}, \ldots, \lambda_{b}\right)$. From 6.4 we see $F^{*} \circ \chi_{\omega}^{s}\left(V_{q}(n)\right)$ is independently variable on the spherical cycles in the image of $\pi_{n+q}(X) \rightarrow H_{n+q}(X ; Z)$. By the universality of $B \bar{\Gamma}_{S L_{q}}$ this proves 6.1.

To establish Proposition 6.3 we must show $\chi_{\omega}^{s}\left(y_{2}\right): \pi_{6}\left(B \bar{\Gamma}_{S L_{3}}\right) \rightarrow \mathbf{R}$ is onto, and for this we start with an example. Let $S^{3}$ have a volume form $\tilde{\omega}_{\lambda}$ with $\left\langle\left[\tilde{\omega}_{\lambda}\right],\left[S^{3}\right]\right\rangle=\lambda$. Define an $S L_{3}$-foliation $\left(\mathscr{F}, \omega_{\lambda}\right)$ on $S O_{4}$ by the fibration $p: S O_{4} \rightarrow S^{3}$ and lifting $\tilde{\omega}_{\lambda}$ to $\omega_{\lambda}$. The normal bundle to $\mathscr{F}$ satisfies $Q \cong p^{*} T S^{3}$, where $S O_{4}$ can be identified with the bundle of orthonormal frames of $T S^{3}$. Hence, there is a canonical framing $s$ of $Q$. We claim $\chi_{\omega_{\lambda}}^{s}\left(y_{2}\right) \in H^{6}\left(S_{4}\right)$ is nonzero and proportional to $\lambda$. One way to see this is to note that $\mathscr{F}$ is a foliation by compact leaves and that Proposition 3.2 yields

$$
\int_{S O_{4}} \chi_{\omega_{\lambda}}^{s}\left(y_{2}\right)=\int_{S^{3}}\left\{\int_{L=S O_{3}} \chi_{L}^{s}\left(y_{2}\right)\right\} \tilde{\omega}_{\lambda} .
$$

Given a point $x \in S^{3}$ and the proper choice of framing of $T S_{x}^{3}$, the restriction $\left.s\right|_{p^{-1}(x)}: \mathrm{SO}_{3} \rightarrow \mathrm{SO}_{3}$ is the identity map by the definition of $s$. Thus, the leaf class $\chi_{p^{-1}(x)}^{s}\left(y_{2}\right) \in H^{3}\left(S_{3}\right)$ is a nonzero class which is independent of $x$. Therefore, $\left\langle\chi_{\omega_{\lambda}}^{s}\left(y_{2}\right),\left[S O_{4}\right]\right\rangle=\left\langle\left[\tilde{\omega}_{\lambda}\right],\left[S^{3}\right]\right\rangle \cdot\left\langle\chi_{L}^{s}\left(y_{2}\right),\left[S O_{3}\right]\right\rangle$ is nonzero and proportional to $\lambda$.

Now lift $\left(\mathscr{F}, \omega_{\lambda}\right)$ to the double cover $\mathrm{Spin}_{4}$ of $\mathrm{SO}_{4}$. This produces a new $S L_{3}$-foliation $\left(\mathscr{F}^{\prime}, \omega_{\lambda}^{\prime}\right)$ for which $\chi_{\omega_{\lambda}^{\prime}}^{s}\left(y_{2}\right)$ varies, and is also defined by a fibration $p^{\prime}$ : Spin $_{4} \rightarrow S^{3}$ with fiber $\operatorname{Spin}_{3} \cong S^{3}$. Recall that $p^{\prime}$ admits a section and so $\operatorname{Spin}_{4} \cong S^{3}$ $\times S^{3}$. Clearly, the volume form $\omega_{\lambda}^{\prime}$ evaluates to give zero on the fiber $S^{3}$ of $p^{\prime}$, so the composition $S^{3} \rightarrow B \bar{\Gamma}_{S L_{3}}$ in the diagram

$$
\begin{array}{cccc}
S^{3} \rightarrow \operatorname{Spin}_{4} & \stackrel{f_{\lambda}^{s}}{\rightarrow} & B \bar{\Gamma}_{S L_{3}} \\
p^{\prime} \downarrow & & \downarrow \\
S^{3} & \rightarrow & B \Gamma_{S L_{3}}
\end{array}
$$

is homotopic to a constant.

Attach a 4-cell $e_{4}$ via $\partial e_{4}=S^{3} \cong \operatorname{Spin}_{3}$ to a fiber of $p^{\prime}$ to obtain $X=\operatorname{Spin}_{4} \cup_{S^{3}} e^{4}$ which is homotopic to $S^{3} \vee S^{6}$. The above remarks imply $f_{\lambda}^{s}$ extends to $F_{\lambda}$ : $X \rightarrow B \bar{\Gamma}_{S L_{3}}$ with $F_{\lambda}^{*} \circ \chi_{\omega}^{s}\left(y_{2}\right)$ nonzero and proportional to $\lambda$. Therefore, $\chi_{\omega}^{s}\left(y_{2}\right) \in$ $H^{6}\left(B \bar{\Gamma}_{S L_{3}}\right)$ varies on the spherical cycles in the images of $\left(F_{\lambda}\right)_{\#}: \pi_{6}(X) \rightarrow \pi_{6}\left(B \bar{\Gamma}_{S L_{3}}\right)$.

Theorem 6.1 and Proposition 6.3 have wide-ranging consequences for the higher homotopy groups $\pi_{*}\left(B \bar{\Gamma}_{S L_{q}}\right)$. To state these results it is necessary to introduce some new ideas. Applications of the following will be given in $\$ 7$.

The elements in the image of $\sigma: \operatorname{Span}_{\mathbf{R}} V_{q}(n) \rightarrow \pi_{n+q}\left(B \bar{\Gamma}_{S L_{q}}\right) \otimes \mathbf{Q}$ are detected by the cohomology classes $\chi_{\omega}^{s}\left(V_{q}(n)\right) \subseteq H^{q+n}\left(B \bar{\Gamma}_{S L_{q}}\right)$. These classes are represented by 
cocycles $\left\{\Delta\left(y_{I}\right) \wedge \omega\right\}$ which have the property that their products vanish as cocycles, $\Delta\left(y_{I}\right) \omega \wedge \Delta\left(y_{I^{\prime}}\right) \omega=0$, since $\omega \wedge \omega=0$ for the volume form $\omega$. It then follows easily from rational homotopy theory that the subset $\cup_{n<q} \sigma\left(V_{q}(n)\right) \subseteq \pi_{*}\left(B \bar{\Gamma}_{S L_{q}}\right) \otimes$ $\mathbf{Q}$ generates a free, graded Lie subalgebra. These ideas are presented elegantly by Haefliger in [12], whose notation we adopt here.

Given a graded vector space $V$, let $\Sigma^{k} V$ denote the $k$-fold suspension: $\Sigma^{k} V$ is isomorphic to $V$ as a vector space, but each $a \in V$ is raised in degree by $k$ to give $\Sigma^{k} a \in \Sigma^{k} V$. We set $\Sigma=\Sigma^{\prime}$.

Recall that $V_{q}(n)$ is the set of elements of degree $n$ in the set $V_{q}$ of Theorem 5.2. Set $V_{q}(0)=\{1\}$, where 1 has degree 0 . For $q \geqslant 2$ define graded vector spaces:

$$
\widetilde{T}_{2}=\sum \operatorname{Span}_{\mathbf{R}} V_{2}(0), \quad \Upsilon_{3}=\bigoplus_{n=0.3} \sum{ }^{2} \operatorname{Span}_{\mathbf{R}} V_{3}(n) \cong \sum{ }^{2} \operatorname{Span}_{\mathbf{R}}\left\{1, y_{2}\right\}
$$

and for $q>3$,

$$
\mathcal{\Psi}_{q}=\bigoplus_{0 \leqslant n<q} \sum^{q-1} \operatorname{Span}_{\mathbf{R}} V_{q}(n)
$$

Let $\mathbf{L} \mathcal{T}_{q}$ denote the free graded Lie algebra over $\mathbf{R}$ generated by the graded vector space $\mathscr{V}_{q}$. For details on the construction of this space, see [12]. If the real dimension of $\mathscr{V}_{q}$ is greater than one, then $\mathbf{L} \mathscr{V}_{q}$ is an infinite-dimensional real vector space which is finite dimensional in each degree. There is a natural inclusion $\mathcal{V}_{q} \rightarrow \mathbf{L} \mathcal{V}_{q}$ as a Lie algebra basis, from which we derive an inclusion $\Sigma \mathcal{V}_{q} \subseteq \Sigma \mathbf{L} \mathcal{V}_{q}$. Theorem 6.1 and Proposition 6.3 assert that for all $q \geqslant 2$, the $\mu$-classes in $\chi_{\omega}^{s}\left(V_{q}\right)$ define a degree preserving epimorphism $\pi_{*}\left(B \bar{\Gamma}_{S L_{q}}\right) \rightarrow \Sigma \mathcal{W}_{q}$ onto a real graded vector space, and $\sigma$ : $\sum \mathcal{V}_{q} \rightarrow \pi_{*}\left(B \bar{\Gamma}_{S L_{q}}\right) \otimes \mathbf{Q}$ is a rational section. There exist extensions of these maps which yield the following general results.

THEOREM 6.5. Let $q \geqslant 2$.

(a) There is a natural, degree preserving group homomorphism onto a real graded vector space $\pi_{*}\left(B \bar{\Gamma}_{S L_{q}}\right) \rightarrow \Sigma \mathbf{L} \mathcal{V}_{q}$.

(b) There is a degree preserving inclusion of a real vector space $\sigma: \Sigma \mathbf{L} \mathcal{V}_{q} \rightarrow$ $\pi_{*}\left(B \bar{\Gamma}_{S L_{q}}\right) \otimes \mathbf{Q}$ and $\boldsymbol{\sigma}$ is a rational section of the map in (a).

The homomorphism in 6.5(a) is defined using the dual homotopy invariants associated to the $\mu$-classes. The dual homotopy invariants corresponding to the secondary classes of an $S L_{q}$-foliation are developed in [17]; this theory can be adapted in a straightforward way to produce invariants of the dga map $\Sigma^{q} \chi_{\omega}^{s}$ : $\sum^{q} \Lambda\left(y_{2}, \ldots, y_{q}\right) \rightarrow \mathbb{Q}(M)$ associated to a framed $S L_{q}$-foliation $(\mathscr{F}, \omega, s)$ on $M$. The proof of Theorem 6.5 then proceeds in the same fashion as that of Proposition 5.13 in [18]. However, to give the proof here would take us too far afield, and so it is omitted.

We conclude this section with a partial description of the sets $\Sigma \mathbf{L} \mathcal{V}_{q}$ for small values of $q$. The numbers after the spanning sets are the respective degrees of the 
corresponding basis elements of $\Sigma \mathbf{L} \mathcal{V}_{q}$ :

$q=2, \quad \sum \mathbf{L} \mathcal{V}_{2}=\operatorname{Span}_{\mathbf{R}}\{[\omega],[\omega, \omega]\} ;$ degrees 2, 3,

$q=3, \quad \sum \mathbf{L} \mathcal{V}_{3}=\operatorname{Span}_{\mathbf{R}}\left\{[\omega],\left[y_{2} \omega\right],\left[y_{2} \omega, \omega\right],\left[\omega,\left[y_{2} \omega, \omega\right]\right],\left[y_{2} \omega, y_{2} \omega\right], \ldots\right\} ;$

degrees $3,6,8,10,11, \ldots$,

$q=4, \quad \sum \mathbf{L} \mathscr{V}_{4}=\operatorname{Span}_{\mathbf{R}}\left\{[\omega],\left[y_{2} \omega\right],[\omega, \omega],\left[y_{2} \omega, \omega\right],[\omega,[\omega, \omega]], \ldots\right\} ;$

degrees $4,7,7,10,10, \ldots$.

The next qualitative change occurs for

$$
\begin{array}{r}
q=8, \quad \sum \mathbf{L} \mathcal{V}_{8}=\operatorname{Span}_{\mathbf{R}}\left\{[\omega],\left[y_{2} \omega\right],\left[y_{4} \omega\right],[\omega, \omega],\left[y_{2} \omega, \omega\right],\right. \\
\left.[\omega,[\omega, \omega]],\left[y_{4} \omega, \omega\right],\left[y_{2} \omega, y_{4} \omega\right],\left[\omega,\left[y_{2} \omega, \omega\right]\right], \ldots\right\} ; \\
\text { degrees } 8,11,15,15,18,22,22,25,25, \ldots
\end{array}
$$

7. The local homology of $\operatorname{Diff}_{\omega}^{c} \mathbf{R}^{q}$. The calculations of $\pi_{*}\left(B \bar{\Gamma}_{S L_{q}}\right)$ in $\S 6$ and the theorems of McDuff for $S L_{q}$-foliations generalizing the Mather-Thurston Theorem enable us to make many calculations of the local homology groups $H_{*}\left(B \overline{\operatorname{Diff}}_{\omega}^{c} \mathbf{R}^{q} ; \mathbf{Q}\right)$.

Let $X$ be a connected manifold of dimension $q$ with no boundary and endowed with a volume form $\omega$. If $X$ is open, we assume each end of $X$ has infinite $\omega$-volume. For technical convenience, we also assume $H^{q-1}(X)=0$; see [25] for the details of the general case. Recall that $\operatorname{Diff}_{\omega} X$ denotes the group of diffeomorphisms of $X$ which preserve $\omega$, and $\operatorname{Diff}_{\omega}^{c} X$ is the subgroup of diffeomorphisms which are compactly supported. We let $G$ denote either of these two groups, endowed with the compact-open topology; $G^{\delta}$ denotes the group $G$ with the discrete topology and $\bar{G}$ is the homotopy fiber of $G^{\delta} \rightarrow G$. Note that $\bar{G}$ is again a group.

The homology group $H_{*}(B \bar{G} ; Z)$ depends only on the structure of $G$ in a neighborhood of the identity and so is called the local homology of $G$ [28].

It is shown in [24] that $B \bar{G} \times X$ has a natural $S L_{q}$-foliation transverse to the fibers of the projection onto $B \bar{G}$. Thus, there is a homotopy commutative diagram

$$
\begin{array}{ccc}
B \bar{G} \times X & \stackrel{f}{\rightarrow} & B \Gamma_{S L_{q}} \\
\downarrow & \gamma \nearrow & \downarrow \nu \\
X & \stackrel{\tau}{\rightarrow} & B S L_{q}
\end{array}
$$

where $\tau$ classifies the tangent bundle to $X$ and $f$ is the classifying map for the $S L_{q}$-foliation on $B \bar{G} \times X$. There is a lifting $\gamma$ of $\tau$ which classifies the point foliation on $X$.

Let $L \rightarrow X$ be the pull-back under $\tau$ of the fibration $\nu: B \Gamma_{S L_{q}} \rightarrow B S L_{q}$, with fiber $B \bar{\Gamma}_{S L_{q}}$. From diagram (7.1) and adjunction we obtain a map $f_{X}: B \bar{G} \rightarrow \mathcal{S}(X)$, where $\delta(X)$ is the component of the space of sections of $L \rightarrow X$ which contains the map $\gamma$. If $G=\operatorname{Diff}_{\omega}^{c} X$, then we can further choose the map $f_{X}$ to have image in the space $\delta_{c} X$ of sections which are equal to $\gamma$ outside a compact set in $X$.

For example, $\delta_{c}\left(\mathbf{R}^{q}\right)$ is homotopy equivalent to the component $\Omega^{q}\left(B \Gamma_{S L_{q}}\right)_{0}$ of $\Omega^{q}\left(B \Gamma_{S L_{q}}\right)$ which contains the base point, and so $\delta_{c}\left(\mathbf{R}^{q}\right) \simeq \Omega^{q}\left(B \bar{\Gamma}_{S L_{q}}\right)_{0} \simeq \Omega^{q} B \Gamma_{S L_{q}}$. 
When $X$ is a compact manifold with trivial tangent bundle, then $\delta(X) \cong \delta_{c}(X) \simeq$ $\operatorname{Maps}\left(X, B \bar{\Gamma}_{S L_{q}}\right)_{0}$, the latter space being the set of continuous maps which are not necessarily base-point preserving.

In a sequence of papers [24-26], McDuff has proven a generalization of the Mather-Thurston Theorem [28] which applies to $S L_{q}$-foliations. We state this theorem in the special case where the flux homomorphism is zero.

THEOREM 7.2 (MCDUfF). Let $X$ be a manifold without boundary and $\omega$ a volume form on $X$ so that each end of $X$ has infinite w-volume if $X$ is open. Assume that $H^{q-1}(X)=0$. Then the map $f_{X}$ defined above induces isomorphisms

$$
\begin{aligned}
& f_{X^{*}}: H_{*}\left(B \overline{\operatorname{Diff}}_{\omega} X ; Z\right) \rightarrow H_{*}(\delta(X) ; Z), \\
& f_{X^{*}}: H_{*}\left(B \overline{\operatorname{Diff}}_{\omega}^{c} X ; Z\right) \rightarrow H_{*}\left(\delta_{c}(X) ; Z\right) .
\end{aligned}
$$

For $q>2$, Thurston and Banyaga (see $[1,32]$ ) have shown by geometric arguments that $H_{1}\left(B \overline{\operatorname{Diff}}_{\omega}^{c} \mathbf{R}^{q} ; Z\right)=0$. Therefore, $H_{1}\left(\Omega^{q} B \bar{\Gamma}_{S L_{q}} ; Z\right) \cong H_{1}\left(\delta_{c}\left(\mathbf{R}^{q}\right)\right) \cong 0$ which implies $\pi_{q+1}\left(B \bar{\Gamma}_{S L_{q}}\right)=0$. From this we can conclude that the mapping spaces $\delta(X)$ and $\delta_{c}(X)$ are simply connected. For degrees $n>1$, Theorem 7.2 reduces the calculation of $H_{n}\left(B \overline{\operatorname{Diff}}_{\omega}^{c} X ; Z\right)$ to that of the homology of a space of sections or maps. Using the results of $\S 6$ on the nontriviality of $\pi_{*}\left(B \bar{\Gamma}_{S L_{q}}\right)$, it is in theory possible to make extensive calculations of these homology groups using Sullivan's model for the space of sections of a fibration [34]. We consider in this paper only the case $X=\mathbf{R}^{q}$ and $\omega=d x_{1} \wedge \cdots \wedge d x_{q}$ the standard volume form. Then by 7.2 , there are isomorphisms

$$
\begin{aligned}
& H_{*}\left(B \overline{\operatorname{Diff}}_{\omega} \mathbf{R}^{q} ; Z\right) \cong H_{*}\left(B \bar{\Gamma}_{S L_{q}} ; Z\right), \\
& H_{*}\left(B \overline{\operatorname{Diff}}_{\omega}^{c} \mathbf{R}^{q} ; Z\right) \cong H_{*}\left(\Omega^{q} B \overline{\bar{\Gamma}}_{S L_{q}} ; Z\right) .
\end{aligned}
$$

The results of $\$ 5$ on nontrivial cohomology invariants from the $\mu$-classes all apply to $\overline{\operatorname{Diff}}_{\omega} \mathbf{R}^{q}$, and the homotopy results of $\S 6$ can be applied to study $\overline{\operatorname{Diff}}_{\omega}^{c} \mathbf{R}^{q}$. Our interest is this latter group.

By the Bott-Samelson Theorem, the rational homology $H_{*}\left(\Omega^{q} B \overline{\bar{\Gamma}}_{S L_{q}} ; \mathbf{Q}\right)$ is an exterior algebra with generators the primitives in the Hopf algebra structure. The space of primitives can be identified with $\pi_{*}\left(\Omega^{q} B \overline{\bar{\Gamma}}_{S L_{q}}\right) \otimes \mathbf{Q} \cong \pi_{*+q}\left(B \overline{\bar{\Gamma}}_{S L_{q}}\right) \otimes \mathbf{Q}$. Let $\Sigma^{-q} V$ denote the $q$-fold desuspension of the graded vector space $V$. Then Theorem 7.2 implies

COROLLARY 7.3. There is a natural isomorphism of graded rational vector spaces

$$
\Lambda_{\mathbf{Q}}\left\{\Sigma^{-q} \pi_{*}\left(B \overline{\bar{\Gamma}}_{S L_{q}}\right) \otimes Q\right\} \stackrel{\cong}{\rightarrow} H_{*}\left(B \overline{\operatorname{Diff}}_{\omega}^{c} \mathbf{R}^{q} ; \mathbf{Q}\right) .
$$

At the end of $\S 6$ the real graded Lie algebra $\mathbf{L} \mathcal{V}_{q}$ was introduced, and Theorem 6.5 asserts there is an inclusion of rational vector spaces $\sigma: \Sigma \mathbf{L} \widetilde{V}_{q} \rightarrow \pi_{*}\left(B \bar{\Gamma}_{S L_{q}}\right) \otimes \mathbf{Q}$. Recall that the space $\Sigma \mathbf{L} \mathcal{V}_{q}$ contains one generator of degree $q$, corresponding to the class $[\omega]$ in $\pi_{q}\left(B \bar{\Gamma}_{S L_{q}}\right) \otimes \mathbf{R}$, and $\Sigma \mathbf{L} \mathcal{V}_{q}$ has no elements of degree less than $q$. Define a new graded vector space $\Sigma_{0} \mathbf{L} \mathcal{V}_{q}$ which is equal to $\Sigma \mathbf{L} \mathcal{V}_{q}$ in degrees greater than $q$, but 
has no elements in degree $q$. Since $\pi_{m}\left(B \overline{\bar{\Gamma}}_{S L_{q}}\right) \cong \pi_{m}\left(B \bar{\Gamma}_{S L_{q}}\right)$ for $m>q$ we thus obtain an inclusion of rational vector spaces $\sigma_{0}: \Sigma_{0} \mathbf{L} \mathcal{T}_{q} \rightarrow \pi_{*}\left(B \bar{\Gamma}_{S L_{q}}\right) \otimes \mathbf{Q}$. Combining this with Corollary 7.3 we obtain

THEOREM 7.4. There is a natural inclusion of graded rational vector spaces

$$
\Sigma^{-q}\left(\sum_{0} \mathbf{L} \mathcal{T}_{q}\right)^{\Sigma^{-1} \sigma_{0}} H_{*} j\left(B \overline{\operatorname{Diff}_{\omega}^{c}} \mathbf{R}^{q} ; \mathbf{Q}\right)
$$

for all $q \geqslant 2$. When $q \geqslant 3, \Sigma^{-q}\left(\Sigma_{0} \mathbf{L}_{q}\right)$ is a real vector space with infinite real dimension.

The image of $\Sigma^{-q} \sigma_{0}$ is contained in the space of primitives of $H_{*}\left(B \overline{\operatorname{Diff}_{\omega}^{c}} \mathbf{R}^{q} ; \mathbf{Q}\right) \cong$ $H_{*}\left(\Omega^{q} B \bar{\Gamma}_{S L_{q}} ; \mathbf{Q}\right)$ so one can extend 7.4 to yield

COROLlaRY 7.5. There is an inclusion of the free exterior algebra over $\mathbf{Q}$ with basis the real vector space $\Sigma^{-q}\left(\Sigma_{0} \mathbf{L} \mathcal{T}_{q}\right)$,

$$
\Lambda_{\mathbf{Q}}\left(\Sigma^{-q}\left(\sum_{0} \mathbf{L}_{q}\right)\right) \stackrel{\Lambda \Sigma^{-1} \sigma_{0}}{\rightarrow} H_{*}\left(B \overline{\operatorname{Diff}_{\omega}^{c}} \mathbf{R}^{q} ; \mathbf{Q}\right)
$$

The simplest case of this corollary can be stated as

Proposition 7.6. For all $q \geqslant 3$ there is an inclusion $\sigma_{0}: \mathbf{R} \rightarrow H_{3}\left(B \overline{\operatorname{Diff}_{\omega}^{\prime}} \mathbf{R}^{q} ; \mathbf{Q}\right)$.

This shows the Thurston-Banyaga vanishing theorem for $H_{1}\left(B \overline{\operatorname{Diff}_{\omega}^{c}} \mathbf{R}^{q} ; Z\right)$ does not extend to degree 3 or above. It is unknown whether the group $H_{2}\left(B \overline{\operatorname{Diff}_{\omega}^{c}} \mathbf{R}^{q} ; \mathbf{Q}\right)$ vanishes.

We remark that the rational vector space $\Lambda_{\mathbf{Q}}\left(\Sigma^{-q}\left(\Sigma_{0} \mathbf{L} \mathcal{V}_{q}\right)\right)$ is incredibly large by almost any standard. Even for $q=2, \Sigma^{-2}\left(\Sigma_{0} \mathbf{L} \Upsilon_{2}\right)$ is a real vector space with basis element $\Sigma^{-2}[\omega, \omega]$ of degree 1 , so $H_{*}\left(B \overline{\operatorname{Diff}}{ }_{\omega}^{c} \mathbf{R}^{2} ; \mathbf{Q}\right)$ contains the rational graded exterior algebra $\Lambda_{\mathbf{Q}} \mathbf{R}$, where each nonzero element of $\mathbf{R}$ has degree one.

It is natural to ask what properties of the group $\overline{\operatorname{Diff}_{\omega}^{c}} \mathbf{R}^{q}$ give rise to these homology classes. For example, one asks for invariants of $\overline{\operatorname{Diff}}_{\omega}^{c} \mathbf{R}^{q}$ which can detect a nontrivial homology class in the image of $\Sigma^{-q} \sigma_{0}$. This has been answered by McDuff for one of the classes when $q$ is even, that being $\Sigma^{-q}[\omega, \omega]$ of degree $(q-1)$. We restrict to the case $q=2$. Then $\eta \equiv \Sigma^{-2}[\omega, \omega]$ is a basis for $\Sigma^{-2}\left(\Sigma_{0} \mathbf{L} \mathcal{V}_{2}\right)$ as a real vector space, and we have $\Sigma^{-2} \sigma_{0}(\eta) \in H_{1}\left(B \overline{\operatorname{Diff}_{\omega}^{c}} \mathbf{R}^{2} ; \mathbf{Q}\right)$ is nonzero. McDuff shows in [27] that this element is detected in a natural way by the Calabi invariant in $H^{1}\left(B \overline{\operatorname{Diff}}_{\omega}^{c} \mathbf{R}^{2} ; \mathbf{R}\right)$. This correspondence is a basis for hope that other classes in the image of $\Sigma^{-q} \sigma_{0}$ are detected by natural invariants of groups of volume-preserving diffeomorphisms. A further discussion of the Calabi invariant and related ideas can be found in Banyaga [37].

\section{REFERENCES}

1. A. Banyaga, Sur la structure du groupe des diffeomorphisms qui préservent une form symplectic, Comment. Math. Helv. 53 (1978), 174-227.

2. H. J. Baues and J. M. Lemaire, Minimal models in homotopy theory, Math. Ann. 225 (1977), 219-242.

3. A. Borel, Stable real cohomology of arithmetic groups, Ann. Sci. École Norm. Sup. 7 (1974), 235-272.

4. R. Bott, Lectures on characteristic classes and foliations, Lecture Notes in Math., vol. 279 , Springer-Verlag, Berlin and New York, 1972, pp. 1-94. 
5. On the characteristic classes of groups of diffeomorphisms, Enseign. Math. 23 (1977), $208-220$

6. R. Bott and A. Haefliger, On characteristic classes of $\Gamma$-foliations, Bull. Amer. Math. Soc. 78 (1972), $1038-1044$.

7. A. Connes, Sur la théorie non-commutative de l'integration, Lecture Notes in Math., vol. 725, Springer-Verlag, Berlin and New York, 1979, pp. 19-143.

8. A. Connes and G. Skandalis, Théorème de l'indice pour les feuilletages, C.R. Acad. Sci. Paris Sér. I Math. 292 (1981), 871-876.

9. G. Duminy, L'invariant de Godbillon-Vey d'un feuilletage se localise dans les feuilles ressort, preprint, 1982.

10. A. Haefliger, Homotopy and integrability, Lecture Notes in Math., vol. 197, Springer-Verlag, Berlin and New York, 1971, pp. 133-166.

11. _ Differentiable cohomologv, Course given at Varenna, Italy, August 1976.

12. Whitehead products and differential forms, Lecture Notes in Math., vol. 652, Springer-Verlag, Berlin and New York, 1978, pp. 13-24.

13. J. Heitsch, Independent variation of secondary classes, Ann. of Math. (2)108 (1978), 421-460.

14. __ Flat bundles and residues for foliations, Invent. Math. (to appear).

15. J. Heitsch and S. Hurder, Secondary classes, Weil operators and the geometry of foliations, preprint, 1982.

16. M. Hirsch and W. Thurston. Foliated bundles, invariant measures and flat bundles, Ann. of Math. (2) 101 (1975), 369-390

17. S. Hurder, Dual homotopy invariants of G-foliations, Topology 20 (1981), 365-387.

18. _ Dual homotopy invariants of $G$-foliations, Thesis, University of Illinois, Urbana, Illinois, 1980

19. On the secondary classes of foliations with trivial normal bundles, Comment. Math. Helv. 56 (1981), 307-326.

20. __ Vanishing of secondary classes for compact foliations, J. London Math. Soc. (to appear).

21. Foliation dyamics and leaf invariants, preprint, 1982.

22. F. Kamber and P. Tondeur, Foliated bundles and characteristic classes, Lecture Notes in Math., vol. 493. Springer-Verlag, Berlin and New York, 1975.

23. C. Lazarov, A permanence theorem for exotic classes, J. Differential Geom. 14 (1979), 475-486

24. D. McDuff, On groups of volume-preserving diffeomorphisms and foliations with transverse volume form, Proc. London Math. Soc. 43 (1981), 295-320.

25. L_ Local homology of groups of volume-preserving diffeomorphisms. I, Ann. Sci. Ećole Norm. Sup. (to appear).

26. _ The local homologv of groups of volume-preserving diffeomorphisms. II, III, preprint, 1982.

27. Some canonical cohomology classes on groups of volume-preserving diffeomorphisms, Trans.

Amer. Math. Soc. 275 (1983), 345-356.

28. J. Mather, Foliations and local homologv of groups of diffeomorphisms, Proceedings of the ICM, Vancouver, 1974

29. J. Plante, $A$ generalization of the Poincare-Bendixson Theorem for foliations of codimension one, Topology 12 (1973), 177-181.

30. __ Foliations with measure preserving holonomy, Ann. of Math. (2) 102 (1975), 327-361

31. D. Ruelle and D. Sullivan, Currents, flows and diffeomorphisms, Topology 14 (1975), 319-327.

32. W. Thurston, On the structure of the group of volume-preserving diffeomorphisms, preprint, 1973.

33. H. Shulman and D. Tischler, Leaf invariants for foliations and the van Est isomorphism, J. Differential Geom. 11 (1976), 535-546.

34. D. Sullivan, Infinitesimal computations in topologv, Inst. Hautes Études Sci. Publ. Math. 47 (1977), 269-331.

35. Cycles for the dynamical study of foliated manifolds and complex manifolds, Invent. Math. 36 (1976), 225-255.

36. R. Edwards, K. Millet and D. Sullivan, Foliations with all leaves compact, Topology 16 (1977), 13-32.

37. A. Banyaga, On fixed points of symplectic maps, Invent. Math. 56 (1980), 215-229.

38. F. Kamber and P. Tondeur, Characteristic invariants of foliated bundles, Manuscripta Math. 11 (1974), 51-89.

Department of Mathematics, Princeton University, Princeton, New Jersey 08544 